\title{
Infrared Colors of the Gamma-ray-detected Blazars
}

\section{Citation}

D'Abrusco, R., F. Massaro, M. Ajello, J. E. Grindlay, Howard A. Smith, and G. Tosti. 2012. "INFRARED COLORS OF THE GAMMA-RAY-DETECTED BLAZARS." The Astrophysical Journal 748 (1): 68. https://doi.org/10.1088/0004-637x/748/1/68.

\section{Permanent link}

http://nrs.harvard.edu/urn-3:HUL.InstRepos:41399787

\section{Terms of Use}

This article was downloaded from Harvard University's DASH repository, and is made available under the terms and conditions applicable to Other Posted Material, as set forth at http:// nrs.harvard.edu/urn-3:HUL.InstRepos:dash.current.terms-of-use\#LAA

\section{Share Your Story}

The Harvard community has made this article openly available.

Please share how this access benefits you. Submit a story.

Accessibility 


\title{
INFRARED COLORS OF THE GAMMA-RAY-DETECTED BLAZARS
}

\author{
R. D’Abrusco ${ }^{1}$, F. Massaro ${ }^{2}$, M. Ajello ${ }^{2}$, J. E. Grindlay ${ }^{1}$, Howard A. Smith ${ }^{1}$, and G. Tosti ${ }^{2,3,4}$ \\ ${ }^{1}$ Harvard-Smithsonian Astrophysical Observatory, 60 Garden Street, Cambridge, MA 02138, USA \\ 2 SLAC National Laboratory and Kavli Institute for Particle Astrophysics and Cosmology, 2575 Sand Hill Road, Menlo Park, CA 94025, USA \\ ${ }^{3}$ Dipartimento di Fisica, Universitadegli Studi di Perugia, 06123 Perugia, Italy \\ ${ }^{4}$ Istituto Nazionale di Fisica Nucleare, Sezione di Perugia, 06123 Perugia, Italy \\ Received 2011 September 16; accepted 2012 January 11; published 2012 March 6
}

\begin{abstract}
Blazars constitute the most enigmatic class of extragalactic $\gamma$-ray sources, and their observational features have been ascribed to a relativistic jet closely aligned to the line of sight. They are generally divided in two main classes: the BL Lac objects (BL Lacs) and the flat-spectrum radio quasars (FSRQs). In the case of BL Lacs the double-bumped spectral energy distribution (SED) is generally described by the synchrotron self-Compton (SSC) emission, while for the FSRQs it is interpreted as due to external Compton (EC) emission. Recently, we showed that in the [3.4]-[4.6]-[12] $\mu \mathrm{m}$ color-color diagram the blazar population covers a distinct region (i.e., the WISE blazar Strip (WBS)) clearly separated from the other extragalactic sources that are dominated by thermal emission. In this paper, we investigate the relation between the infrared and $\gamma$-ray emission for a subset of confirmed blazars from the literature, associated with Fermi sources, for which WISE archival observations are available. This sample is a proper subset of the sample of sources used previously, and the availability of Fermi data is critical to constrain the models on the emission mechanisms for the blazars. We found that the selected blazars also lie on the WBS covering a narrower region of the infrared color-color planes than the overall blazar population. We then found an evident correlation between the IR and $\gamma$-ray spectral indices expected in the SSC and EC frameworks. Finally, we determined the ratio between their $\gamma$-ray and infrared fluxes, a surrogate of the ratio of powers between the inverse Compton and the synchrotron SED components, and used such parameter to test different blazar emitting scenarios.
\end{abstract}

Key words: BL Lacertae objects: general - gamma rays: galaxies - infrared: galaxies - radiation mechanisms: non-thermal

Online-only material: color figures

\section{INTRODUCTION}

Blazars are an intriguing class of active galactic nuclei (AGNs), dominated by non-thermal radiation over the entire electromagnetic spectrum. Their emission extends from radio to $\mathrm{TeV}$ energies with a broadband spectral energy distribution (SED) typically described by two main components, the first peaking from IR to X-ray bands while the second often dominating the $\gamma$-ray energy range in which blazars are the most commonly detected extragalactic sources (e.g., Abdo et al. 2010; Massaro et al. 2011b).

The distinguishing observational properties of blazars also include flat radio spectra, high observed luminosity coupled with rapid variability at all frequencies, and highly variable radio to optical polarization. In particular, they are a dominant class of extragalactic sources at radio, microwave, and $\gamma$-ray frequencies where thermal emission processes do not produce significant amounts of radiation (Giommi et al. 2011). Adopting the blazar classification scheme given in the ROMA-BZCAT catalog ${ }^{5}$ (Massaro et al. 2009, 2010), based on the width of optical spectral lines, blazars usually come in two flavors: the BL Lac objects (BL Lacs) and flat-spectrum radio quasars (FSRQs), with the latter more or less equivalent to the former population except for stronger emission lines, higher radio to optical polarization, and higher redshift. In addition, the high-energy component of blazar SED is usually found in the GeV band for FSRQs, while it can extend to $\mathrm{TeV}$ energy for BL Lacs. Hereafter, we adopt the naming convention of the ROMA-BZCAT catalog, referring to BL Lacs as BZBs and to the FSRQs as BZQs.

\footnotetext{
http://www.asdc.asi.it/bzcat/
}

These extreme features have been interpreted as radiation arising from a relativistic jet closely aligned to the line of sight and emitting continuous, Doppler-boosted spectra (Blandford \& Rees 1978). For both classes of blazars, according to the widely accepted synchrotron self-Compton (SSC) scenario, the lowenergy component is produced by inverse synchrotron emission from highly relativistic electrons, while the high-energy bump can be attributed to inverse Compton scattering of synchrotron photons by the same population of relativistic electrons that produce the synchrotron emission (Marscher \& Gear 1985; Inoue \& Takahara 1996). A different theoretical interpretation of the high-energy $\gamma$-ray bump characteristic of the BZQ broadband emission invokes the inverse Compton emission of seed photons arising from regions external to the jet (e.g., the broad-line region, accretion disk) as emission mechanism for such feature of the SED. This model is known as the external Compton scenario (EC; e.g., Dermer \& Schlickeiser 2002; Cavaliere \& D'Elia 2002).

Recently, we showed that in the [3.4]-[4.6]-[12] $\mu \mathrm{m}$ color-color diagram the blazars, which are dominated by nonthermal emission mechanism, cover a distinct region (hereafter the WISE blazar Strip (WBS)), well separated from the locus of other extragalactic sources which are dominated by the contribution of thermal radiation (Massaro et al. 2011a).

This result has been obtained using the blazars listed in the ROMA-BZCAT catalog. In the IR, we used data from WISE (Wright et al. 2010). The WISE mission observed the sky at $3.4,4.6,12$, and $22 \mu \mathrm{m}$ in 2010 with an angular resolution of $6 . \prime 1,6.4,6.5$, and $12^{\prime \prime} 0$ in the four bands, achieving $5 \sigma$ point source sensitivities of $0.08,0.11,1$, and $6 \mathrm{mJy}$ in unconfused regions on the ecliptic, respectively (Wright et al. 2010). The 
astrometric accuracy of WISE is 0.'50, 0.'26, 0.'26, and 1".4 for the four WISE bands, respectively (Cutri et al. 2011).

A previous attempt to compare the infrared behavior of blazars with normal galaxies in the $J-H-K$ color-color diagram was performed using the Two Micron All Sky Survey (2MASS) archival data (e.g., Chen et al. 2005). However, our new approach has three advantages over the study performed using 2MASS: (1) mid-IR selection is dominated by dusty objects, in particular spiral and starburst galaxies; (2) the blazar population covers a noticeably narrow region in the [3.4]-[4.6]-[12] $\mu \mathrm{m}$ color-color plot that is clearly statistically separated from the locus dominated by other extragalactic sources (Massaro et al. 2011a); and (3) WISE covers a much larger interval of frequencies and reaches to larger wavelengths than 2MASS, yielding a color-color distribution where stars occupy a narrow and well-defined locus.

The Fermi Large Area Telescope (LAT) Collaboration has recently released the Fermi-LAT second source catalog (2FGL; e.g., The Fermi-LAT Collaboration 2011) including about 800 $\gamma$-ray sources associated with blazars to a high level of confidence; 571 of these blazars are also present in the ROMABZCAT. In this paper, we investigate how the infrared emission of blazars is related to their high-energy $\gamma$-ray radiation by crossmatching the sample of ROMA-BZCAT blazars associated with Fermi sources with the WISE data archive (Wright et al. 2010). In Section 2, we present the blazar sample used throughout our investigation. In Section 3, we show how the Fermi-detected blazars lie on the WBS and we determine the distributions of their IR colors. Then, in Section 4, we investigate a possible relation between the blazar IR and $\gamma$-ray emissions, as predicted in the SSC and EC radiative scenarios. In Section 5, we estimate the Compton dominance (CD) parameter for both the BZQs and the BZBs and we also examine the apparent correlation between the CD and the $\gamma$-ray spectral index for BZBs. In Section 6, we focus our analysis on the BL Lac population and its subclasses. Finally, our summary and discussion are given in Section 7.

Throughout this paper, we assume that the spectral indices, $\alpha$, are defined by flux density, $S_{v} \propto v^{-\alpha}$. Unless otherwise stated, we use cgs units.

\section{SAMPLE SELECTION}

We considered all the blazars in the ROMA-BZCAT that have been associated with a Fermi source, as reported in the 2FGL (The Fermi-LAT Collaboration 2011), for a total number of 571 sources (i.e., 330 BZBs and 241 BZQs). The second edition of the ROMA-BZCAT catalog (Massaro et al. 2009) assembles blazars known in the literature and carefully verified by inspection of their multi-wavelength emission. Members of the ROMA-BZCAT catalog are selected on the basis of a set of criteria involving the presence of detection in the radio band down to $1 \mathrm{mJy}$ flux density at $1.4 \mathrm{GHz}(2.1 \mu \mathrm{m})$, the optical identification and availability of an optical spectrum for further spectral classification, and the detection of X-ray luminosity $L_{X} \geqslant 10^{43} \mathrm{erg} \mathrm{s}^{-1}$. Such criteria do not produce a statistically homogeneous or complete sample of blazars because of the spatially uneven distribution and the variable depths of observations available, but provides the largest and most carefully selected sample of confirmed blazars available to date. The selection and spectral classification of blazars can be difficult due to the absence of typical spectral emission features and to the variability of the emission on timescales of a day or even a few hours. In the ROMA-BZCAT, blazars are also classified into three classes, based on the prominence of the emission features in the optical spectra of these sources. The three classes of such classification are: BZB for the BL Lac sources, i.e., AGNs with featureless optical spectra and narrow emission lines; BZQ for FSRQs with optical spectra showing broad emission lines and typical blazar behavior; BZU for blazars of uncertain type, associated with sources with peculiar characteristics but also showing typical traits of the blazars. This spectral classification will be used throughout this paper. The distinction between BZB and BZQ depends on the choice of an arbitrary threshold value of the equivalent width of the emission lines in the optical spectra of the sources.

The 2FLG catalog contains primarily unresolved sources detected in the all-sky Fermi observations obtained throughout the second year of operation. The sources, after detection and the localization in the sky, are assigned an integrated flux in the $100 \mathrm{MeV}-100 \mathrm{GeV}$ energy range, a spectral shape, and a significance parameter $T S$ based on how significantly each source emerges from the background. Only sources with $T S \geqslant 25$, corresponding to a significance of $4 \sigma$, have been included in the catalog. Each of the 1873 2FLG sources has been considered for identification with already known astronomical sources available in the literature on multi-wavelength observations (The Fermi-LAT Collaboration 2011). For 127 of the 2FLG sources firm identifications have been produced (namely, reliable identifications based on synchronous periodic variability of the sources, coincident spatial morphologies for extended sources, or correlated aperiodic variability). The remaining sources have been investigated for association with sources contained in a list of source catalogs based on different multi-wavelength observations. The ROMA-BZCAT catalog is one of the catalogs used for the association of the 2FLG sources, and 571 Fermi sources have been associated with a BZCAT-ROMA blazar.

We selected all blazars in the ROMA-BZCAT reliably associated with a $\gamma$-ray source of the 2FGL catalog. Then, using the more accurate radio position of the ROMA-BZCAT in place of the coordinates from the Fermi catalog, we searched for infrared counterparts of the above blazars in the WISE archive.

The total number of ROMA-BZCAT blazars in the 2FGL footprint falling in the area surveyed by WISE during the first year (corresponding to $57 \%$ of the whole sky) is 332 (mostly due to the incompleteness of the sky coverage of the ROMABZCAT). In order to search for the positional coincidences of blazars in the observed WISE sky, we considered a search radius 2 ". 4, obtained by combining the $1^{\prime \prime}$ error assumed for the radio position reported in the ROMA-BZCAT (Massaro et al. 2009) with the error on the fourth WISE band at $22 \mu \mathrm{m}$ (i.e., 1".4; see also Wright et al. 2010 for more details). Using a conservative approach in our analysis, we only considered sources in the WISE Preliminary Source Catalog (WPSC) ${ }^{6}$ with a minimum signal-to-noise ratio $(\mathrm{S} / \mathrm{N})$ of 7 in at least one of the four infrared bands.

The number of Fermi-BZCAT blazars with a WISE counterpart within the first region of 2".4 is 296, corresponding to $\sim 45 \%$ of the Fermi-WISE blazar sample in the WPSC, detected with a chance probability of $\sim 3 \%$ (see Maselli et al. 2011; Massaro et al. 2011a for more details) We did not find any multiple matches using 2".4 as search radius. We have used only these 296 blazars for our investigation. The remaining 12 sources with no apparent corresponding WISE sources within 2 .' 4 can be associated with at least one source contained in the WISE catalog using a search radius of $12^{\prime \prime}$, but have not been

\footnotetext{
6 http://wise2.ipac.caltech.edu/docs/release/prelim/preview.html
} 
used in our study. By definition, the Fermi-WISE blazar sample is a proper subset of the sample of ROMA-BZCat blazars with WISE counterparts discussed in the previous paper (Massaro et al. 2011a). The table containing the main parameters of the 296 sources of the Fermi-WISE blazar sample is can be found in Appendix B.

\section{THE FERMI BLAZARS AT INFRARED FREQUENCIES}

As recently shown in Massaro et al. (2011a), the blazars, dominated in the infrared by their synchrotron emission, lie in a distinct region of the [3.4]-[4.6]-[12] $\mu \mathrm{m}$ color-color diagram, and appear to be distinctly separated from the rest of the non-synchrotron-dominated sources populating the sky as observed by WISE. We randomly selected 14 not-overlapping regions of $4 \mathrm{deg}^{2}$ each for a total $56 \mathrm{deg}^{2}$ at high Galactic latitude (Massaro et al. 2011a), within the $116 \mathrm{deg}^{2}$ considered in the WPSC (Cutri et al. 2011). We collected all of the 453,420 sources detected by WISE in its first year catalog (hereafter called WISE thermal sources because most of them are dominated by thermal emission in the infrared energy range), having an $\mathrm{S} / \mathrm{N}>7$ in at least one band, a conservative level for the WPSC release to emphasize the catalog reliability ${ }^{3}$ (Cutri et al. 2011). We have not excluded the stars from the sample of generic WISE-detected sources in the color-color diagrams shown in this paper, since at high galactic latitude, the majority of the observed sources are extragalactic with only little contamination from stars, and we have checked that their presence does not negatively affect the conclusions about the separation of the region of color space occupied by Fermidetected blazars.

We built the [3.4]-[4.6]-[12] $\mu \mathrm{m}$ color-color diagrams from the magnitudes reported in the WISE catalog ${ }^{7}$ for all the WISE thermal sources in the $56 \mathrm{deg}^{2}$ area described above, and for all the sources in the Fermi-WISE blazar sample. In Figure 1, we also show the location of different classes of objects and overlaid to five levels' isodensity contours for all the WISE thermal sources in the $56 \mathrm{deg}^{2}$ region. We plot the blazars of the diagram to characterize their infrared emission. Figure 1 shows that the Fermi-WISE blazars lie in an even more confined region than the general WBS shown in Figure 1 of Massaro et al. (2011a). We note that the relative errors for both the infrared colors are less than $10 \%$ for $97 \%$ of the Fermi-WISE blazar sample but less than $5 \%$ for $\sim 85 \%$ of the sources.

The subregion of the WBS occupied by the Fermi-WISE blazars is well defined. Only two "outliers" out of a total number of 296 blazars are visible in this color-color plane: 2FGL J1506.6+0806 and 2FGL J1550.7+0526 (also known as $4 \mathrm{C}+05.64$ or PKS $1548+056)$. While for these two specific sources the possibility of a wrong association in the 2FGL catalog cannot be ruled out, in general a possible explanation for these or other ROMA-BZCAT sources to lie outside of the WBS might be a thermal contribution from their host galaxy that is non-negligible with respect to the non-thermal IR emission. In general, however, the blazars of the Fermi-WISE blazar sample, dominated by synchrotron emission in the IR, are located in distinctly defined regions of the WISE color-color planes all well separated from the other non-synchrotron-dominated sources detected by WISE.

Assuming that the infrared spectrum of the sources in the Fermi-WISE blazar sample can be described by a power law, we derive the relation between the infrared colors and the spectral

\footnotetext{
All WISE magnitudes are in Vega system.
}

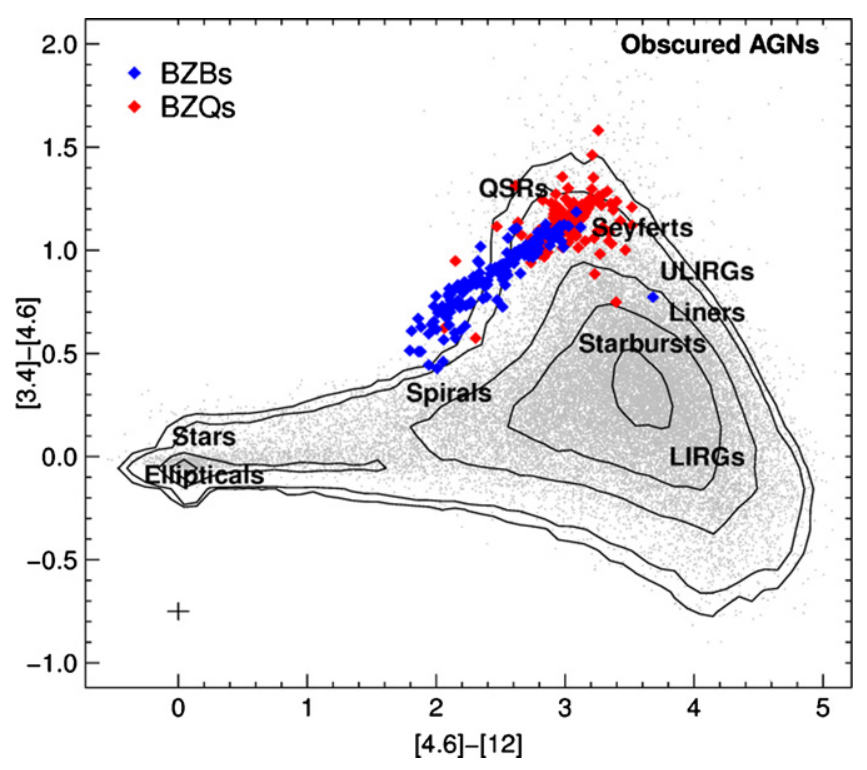

Figure 1. [3.4]-[4.6]-[12] $\mu \mathrm{m}$ color-color diagram of Fermi-WISE blazar sample sources. We plot the 296 blazars associated with a WISE source within a region of radius 2".4. The two blazar classes of BZBs (blue) and BZQs (red) are shown. The background gray dots correspond to 453,420 WISE sources detected in a region of $56 \mathrm{deg}^{2}$ at high Galactic latitude. The isodensity curves for the WISE sources, corresponding to 50, 100, 500, and 2000 sources per unit area in the color-color plane, respectively, are shown (see Section 3). The location of different classes of objects, namely the quasars (QSRs), the ultraluminous infrared galaxies (ULIRGs), and luminous infrared galaxies (LIRGs) is also shown in the plot.

(A color version of this figure is available in the online journal.)

slope $\alpha$. Considering a source of apparent magnitudes $m_{1}, m_{2}$, $m_{3}, m_{4}$ in the four different WISE bands, with the zero-point magnitudes $m_{01}, m_{02}, m_{03}$, and $m_{04}$, respectively, the relation between one color, for example $c_{12}=m_{1}-m_{2}=$ [3.4]-[4.6], and the associated spectral slope $\alpha_{12}$ can be written as

$$
c_{12}=m_{1}-m_{2}=2.5 \alpha_{12} \log \left(\frac{v_{1}}{v_{2}}\right)+\left(m_{01}-m_{02}\right),
$$

where $v_{1}$ and $v_{2}$ are the frequencies corresponding to the 3.4 and $4.6 \mu \mathrm{m}$ wavelengths, respectively. We estimated the values of the spectral indices $\alpha_{12}, \alpha_{23}$, and $\alpha_{34}$ from the three infrared colors $c_{12}, c_{23}$, and $c_{34}$, respectively. Then we compared their distributions to test for the presence of spectral curvature for the sources in the Fermi-WISE blazar sample. We found that the median $\left(1.07,0.94\right.$, and 1.12 for $\alpha_{12}, \alpha_{23}$, and $\left.\alpha_{34}\right)$, the peak values $(1.06,0.99$, and 1.12$)$, and variances $(0.33,0.22$, and 0.26$)$ of the distributions of the three IR spectral indices are consistent with each other. In the following analysis, we will consider the infrared spectral index $\alpha_{\mathrm{IR}}=\alpha_{12}$, because the WISE 3.4 and $4.6 \mu \mathrm{m}$ filters are the most sensitive. Unfortunately, given the WISE-restricted energy range we did not find any hint of a curved spectral shape. In Figure 2, we show the distribution of the spectral indices in each band, $\alpha_{12}, \alpha_{23}$, and $\alpha_{34}$.

Since the sources in the Fermi-WISE blazar sample have been detected in all the four WISE infrared bands, we can also construct the [3.4]-[4.6]-[12]-[22] $\mu \mathrm{m}$ color-color diagram, where the two colors are independent (see Figure 3). In this color-color diagram, the separation between the blazars and the generic WISE sources is even more evident than in the [4.6]-[12]-[22] $\mu \mathrm{m}$ color-color diagram, even though the locus is less narrow than in the case of the [3.4]-[4.6]-[12]-[22] color-color diagram shown in Figure 1). In this plot, we also report the line corresponding to a power-law spectrum of varying 


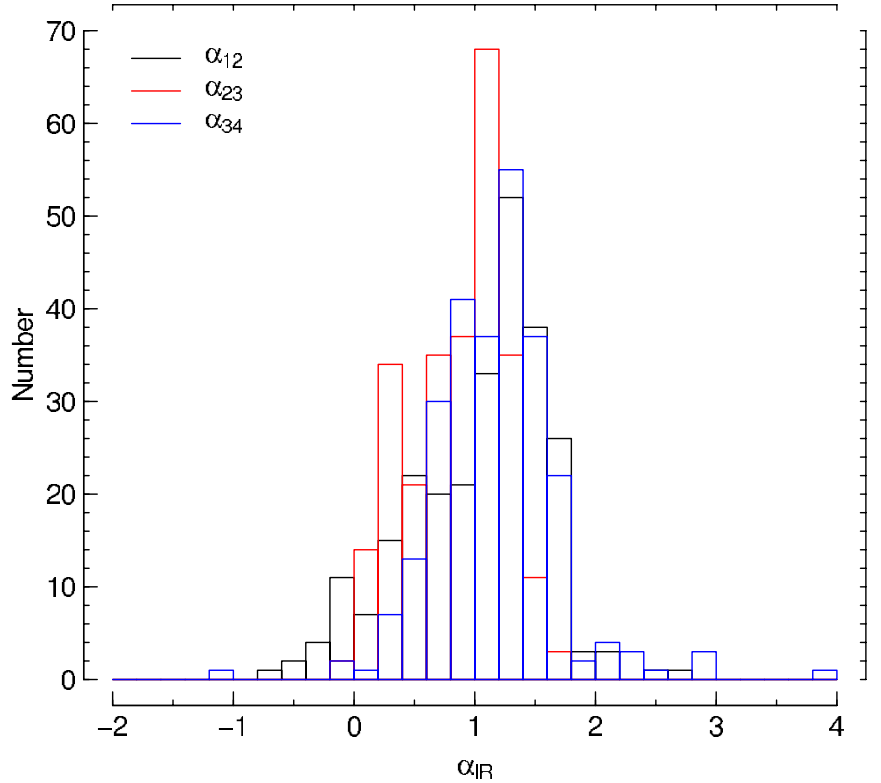

Figure 2. Histograms of the distributions of the infrared spectral indices $\alpha_{12}$, $\alpha_{23}$, and $\alpha_{34}$ for the Fermi-WISE blazar sample, derived by using Equation (1) for the three different colors (see Section 3 for more details).

(A color version of this figure is available in the online journal.)

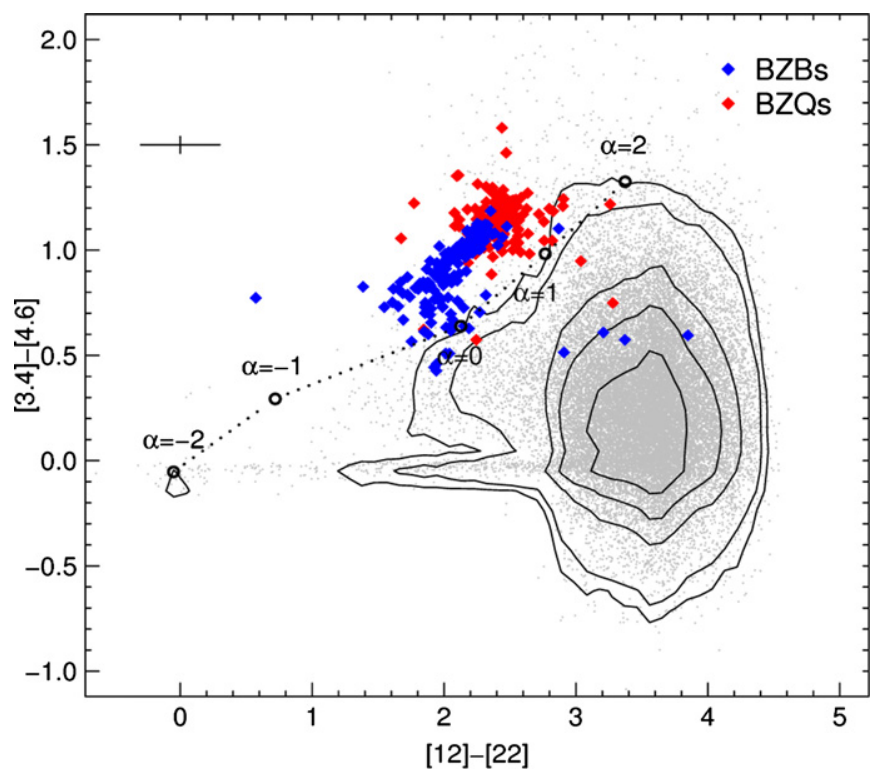

Figure 3. Same as Figure 1 but with a different choice of infrared colors: [3.4]-[4.6]-[12]-[22] $\mu \mathrm{m}$. We also report the black dashed line corresponding to the IR colors generated by a power-law spectrum of spectral index $\alpha_{\mathrm{IR}}$. The black cross shown in the right bottom represents the typical error bars on the infrared colors (see Section 3 for more details).

(A color version of this figure is available in the online journal.)

indices $\alpha_{\mathrm{IR}}$ described by the equation

$$
c_{12}=\left[\frac{\log \left(v_{1} / v_{2}\right)}{\log \left(v_{3} / v_{4}\right)}\left(c_{34}-m_{03}+m_{04}\right)\right]+\left(m_{01}-m_{02}\right),
$$

where $c_{34}=m_{3}-m_{4}=[12]-[22]$ is the infrared color corresponding to the bands at $12 \mu \mathrm{m}$ and $22 \mu \mathrm{m}$, respectively (see Figure 3).

The remaining [4.6]-[12]-[22] $\mu \mathrm{m}$ color-color diagram is shown in Appendix A. In particular, we note that in Figure 3 (and Figure 12 in Appendix A), the regions covered by the Fermi-WISE blazar sample sources are also clearly separated from the thermal WISE sources.

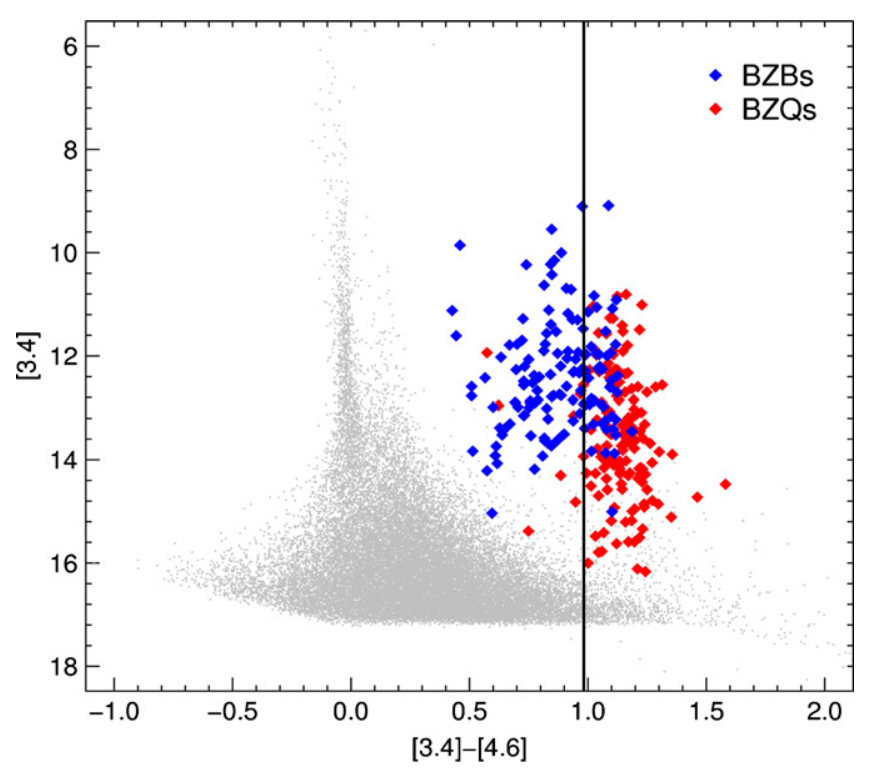

Figure 4. Color-magnitude diagram built with the most sensitive infrared WISE bands, namely the [3.4]-[4.6] color and the [3.4] magnitude. The BZQ sources clearly show the value of the color [3.4]-[4.6] 1 corresponding to a spectral index $\alpha_{\mathrm{IR}}$ of -1 , as a consequence of the WISE observations sampling the peak of their synchrotron components. The black vertical line corresponds to the color value associated with a power law with spectral index $\alpha_{v}=-1$.

(A color version of this figure is available in the online journal.)

Finally, we present a color-magnitude diagram for the three WISE bands with highest sensitivity (Figure 4) where the flux limit of the WISE survey is clearly visible. In this plot, all Fermi-WISE blazar sample sources lie well above the value of the limiting magnitude at $3.4 \mu \mathrm{m}$, and the blazars appear significantly brighter than all the other sources with similar values of the color. Nonetheless, as already discussed for [3.4]-[4.6]-[12]-[22] color-color diagram shown in Figure 1, if on one hand the sources of the Fermi-WISE blazar sample are well separated from the WISE sources even in this color-magnitude plot, on the other hand the region of the plane occupied by the blazars is less compact and well defined than the WBS visible in the [4.6]-[12] $\mu \mathrm{m}$ versus [3.4]-[4.6] $\mu \mathrm{m}$ color-color plane (Figure 1). Other color-magnitude plots of the Fermi-WISE blazar sample are shown in Appendix A.

It is worth stressing that $\sim 95 \%$ of the BZQs have [3.4]-[4.6] color larger than the value of the color associated with a powerlaw spectrum of spectral index 1. This fact suggests that the peaks of the first component (i.e., the synchrotron emission) of these sources occur inside or very close to the WISE spectral range. The situation appears to be different for the BZB class, which displays infrared colors ranging between 0.5 and 1.2 (see Section 6 for more details). It is also interesting to note that all the sources in the Fermi-WISE blazar sample are consistently above the sensitivity limit of the WISE survey, even though this effect is most likely due to the luminosity distribution and selection limits of the Fermi observations.

\section{THE $\alpha_{\mathrm{IR}}-\alpha_{\gamma}$ CORRELATION}

According to the SSC or the EC scenarios, usually adopted to interpret the blazar emission, the particles (i.e., electrons) that are emitting via synchrotron radiation at radio and infrared frequencies are also those that are scattering the photons to high energy, in the X-rays and in the $\gamma$-rays, via inverse Compton emission. Consequently, an empirical correlation between the spectral indices and the fluxes in the infrared and in the $\gamma$-ray 


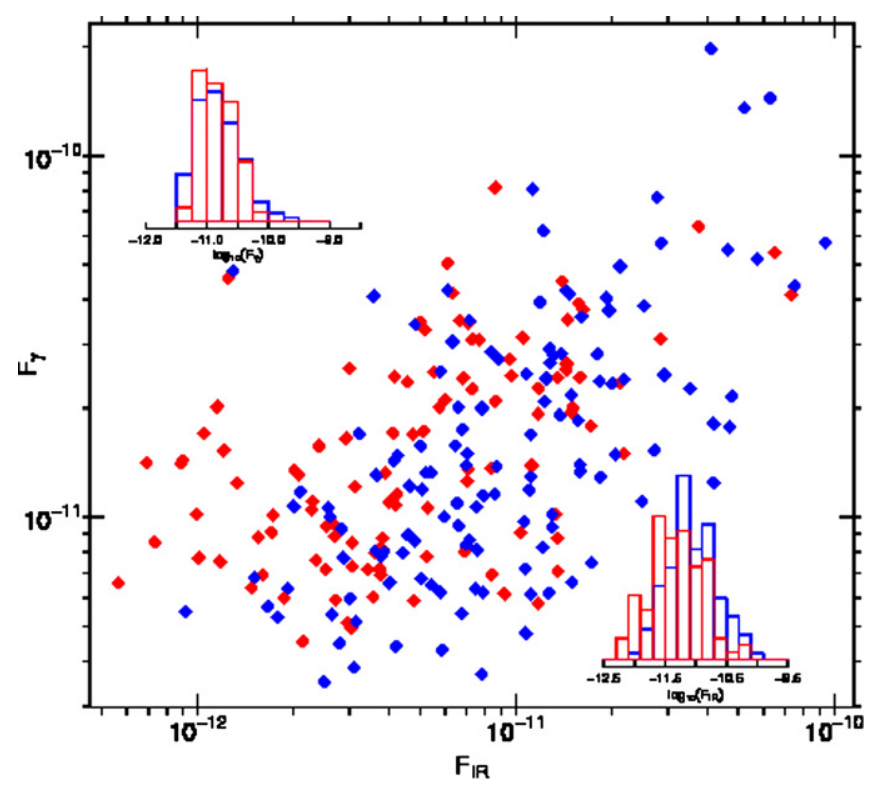

Figure 5. Distribution of the sample of 2FGL detected blazars from the BZCAT-ROMA catalog with WISE counterparts relative to the total $\gamma$-ray flux (from the 2FGL catalog) and the total IR WISE flux calculated from the magnitudes in the four WISE filters. BZBs and BZQs according to the spectral classification available in the ROMA-BZCAT catalog are plotted with red and blue, respectively. The histograms of the IR and $\gamma$-day fluxes distributions for the sample of blazars considered are shown in the two insets of the plot. All fluxes are expressed in cgs units.

(A color version of this figure is available in the online journal.)

energy range is expected, since they originate from the same electron distribution.

The relationships between radio, microwave, and $\gamma$-ray emissions of blazars are discussed by many authors (e.g., Giommi et al. 2011). A positive correlation between the radio and $\gamma$-ray fluxes has been observed, though with large scatter, using several samples (e.g., Kovalev et al. 2009; Giroletti et al. 2010; León-Tavares et al. 2011). However, a correlation between $\gamma$-ray and IR in the spectral range covered by WISE had not been observed to date. This could be mainly due to the lack of $\gamma$-ray observations for a sufficiently large number of blazars, now resolved by the availability of Fermi data. At the same time, the infrared frequencies observed by WISE had not been extensively investigated for blazars despite the fact that the SED frequency peak lies very close to or inside this spectral range, at least for most of the BZQs.

In Figure 5, we show the distribution of the sample of blazars considered in this paper in the plane of the total Fermi energy flux in the $100 \mathrm{MeV}-100 \mathrm{GeV}$ energy interval obtained by spectral fitting in the same range (from the 2FGL catalog), and of the total WISE IR flux derived from the WISE magnitudes. The total IR fluxes have been calculated by summing the fluxes in the four WISE filter obtained as $v f(v)$ and accounting for color corrections as discussed in Wright et al. (2010). BZBs and BZQs are plotted with different colors in this plot. A correlation between $\gamma$-ray and IR fluxes is statistically significant for the whole sample of sources (with correlation coefficient $r_{s}=0.57$ ) and for both classes of BZBs and BZQs separately $\left(r_{s}=0.64\right.$ and $r_{s}=0.61$, respectively).

In Figure 6, we report the $\alpha_{\mathrm{IR}}-\alpha_{\gamma}$ scatterplot with the black line obtained by linear regression on the two spectral indices for BZBs and BZQs together. The associated correlation coefficient is $r_{s}=0.71$, corresponding to a negligible; this implies that the two spectral indices are correlated at a very high

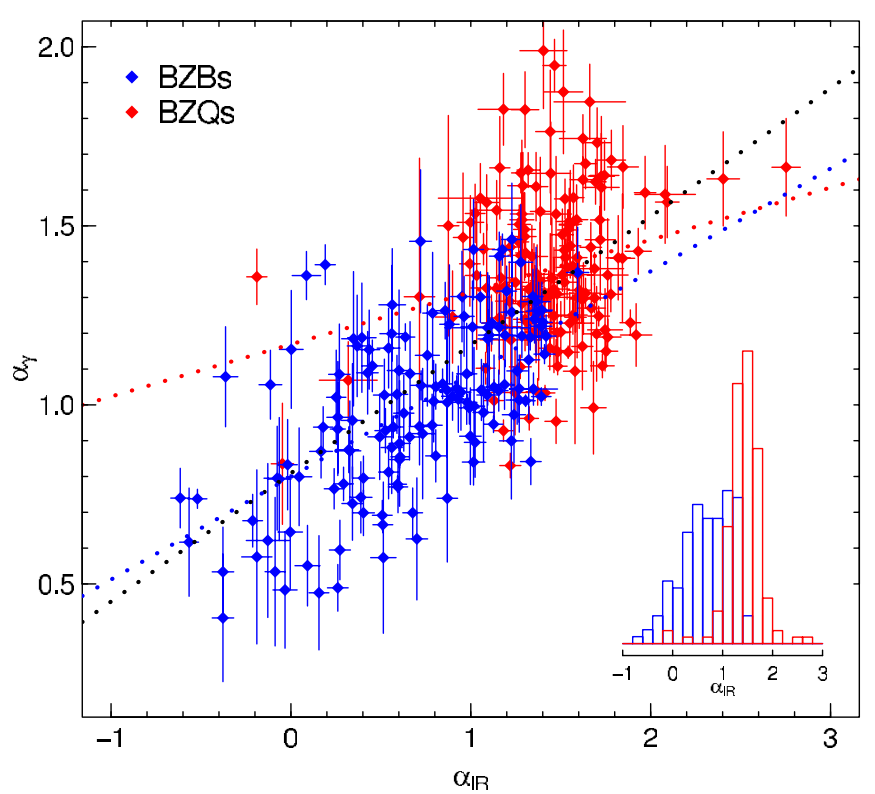

Figure 6. Scatterplot of the $\alpha_{\mathrm{IR}}-\alpha_{\gamma}$ distribution, with the two classes of blazars BZBs (blue) and BZQs (red) shown. In the inset, the histogram of the two distributions of $\alpha_{\mathrm{IR}}$ is plotted, clearly showing the dichotomy between the two classes (see Section 4 for more details). Three linear regression lines with different colors are shown: the black has been obtained by linear fitting on the whole sample of BZBs and BZQs, while the blue and red lines are associated with the regression evaluated for BZBs and BZQs alone, respectively.

(A color version of this figure is available in the online journal.)

level of significance. We have also evaluated the best-fitting linear relations for BZBs and BZQs separately (blue and red lines respectively) and the Spearman's correlation coefficients for these two subsamples of sources. While the correlation between the two spectral indices for the BZBs has a high level of significance with $r_{s}=0.59$ and negligible $p$-value, the correlation for the BZQs is associated with lower values of the correlation coefficient $r_{s}=0.14$, so that the hypothesis that the two parameters are uncorrelated can be rejected at an $80 \%$ level of significance. Overall, the correlation between the IR and $\gamma$-ray spectral indices is dominated by the BZBs. The dichotomy between the BZB and BZQ classes of objects is evident from this plot, not only in the $\alpha_{\gamma}$ distribution and in the comparison of the linear regression lines for the two classes of sources separated (e.g., The Fermi-LAT Collaboration 2011), but also in the $\alpha_{\mathrm{IR}}$ distribution (see the histogram in Figure 6). We performed a Kolmogorov-Smirnov (K-S) test and we found that the distributions of the $\alpha_{\mathrm{IR}}$ for the BZBs and the BZQs differ at $97 \%$ level of significance. We also report in Figure 7 the three-dimensional plot of the two main infrared colors used to build the [3.4]-[4.6]-[12] $\mu \mathrm{m}$ color-color diagram and the $\gamma$-ray spectral index $\alpha_{\gamma}$, to highlight the distinction between the two classes of blazars. The two colors can be here considered as surrogates of the infrared spectral index $\alpha_{\mathrm{IR}}$ (see Section 3 for more details).

\section{THE COMPTON DOMINANCE}

The CD parameter, defined as the ratio between the inverse Compton and synchrotron peak luminosities, can be used to identify the main radiative loss process for the emitting particles, either synchrotron or inverse Compton emission. In particular, since for the majority of the BZQs the WISE and the Fermi spectral ranges are directly sampling or are very close to the peak frequency for both the inverse Compton and 


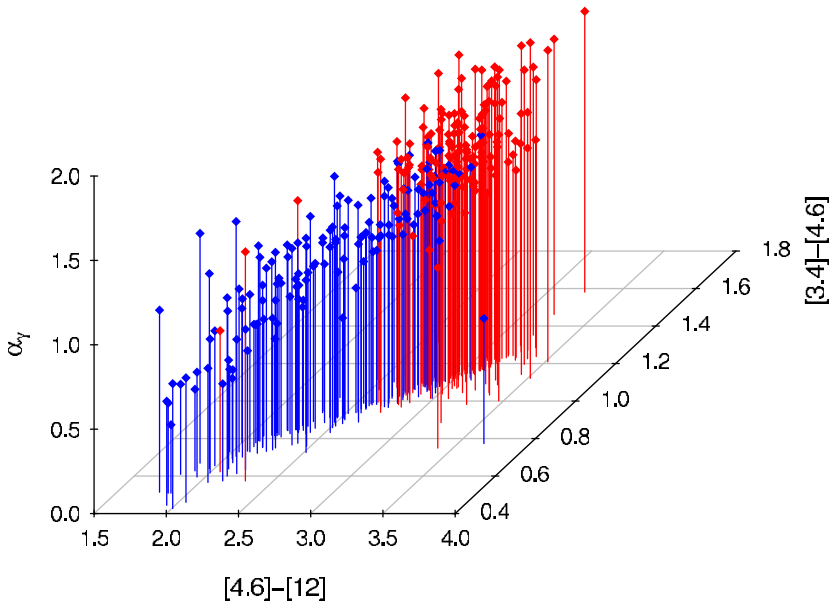

Figure 7. Three-dimensional scatterplot of the distribution of Fermi blazars in the [4.6]-[12]-[3.4]-[4.6]- $\alpha_{\gamma}$ space. The two different classes of blazars, namely the BZBs (blue points) and BZQs (red points), are shown (see Section 4 for more details). The lines extending downward are meant to show the position of the projections of the points on the [3.4]-[46] vs. [4.6]-[12] color-color plane.

(A color version of this figure is available in the online journal.)

the synchrotron components, the ratio between the infrared and the $\gamma$-ray fluxes provides a good estimate of the CD parameter for the Fermi-WISE blazars' sample sources.

In Figure 8, we show the two distributions of the $\mathrm{CD}$ parameter for the different classes of BZBs and BZQs. We performed a K-S test on the CD distributions of BZBs and BZQs and we found that these differ by a $78 \%$ level of significance. Finally, we report the relation between the CD parameter and the $\alpha_{\gamma}$, for both classes of blazars in Figure 9.

\section{THE TWO FAMILIES OF BL LAC OBJECTS}

BL Lacs were originally subclassified into two families on the basis of their radio to X-ray spectral index (Padovani \& Giommi 1995). This classification scheme has been recently extended to all types of non-thermal-dominated AGNs (Abdo et al. 2010), on the basis of the position of the peak of the first SED component, generally assumed to be synchrotron emission. This gives rise to the distinction between the "Low"-"Intermediate"-"High" Synchrotron peaked non-thermal sources (LSPs, ISPs, HSPs), whenever the peak of the synchrotron component lies below $10^{14} \mathrm{~Hz}(\sim 3 \mu \mathrm{m})$, between $10^{14} \mathrm{~Hz}$ and $10^{15} \mathrm{~Hz}(\sim 0.3 \mu \mathrm{m})$, or higher than $10^{15} \mathrm{~Hz}$, respectively (Abdo et al. 2010). Even if blazars should most appropriately be classified on the basis of a complete SED, built with simultaneous data, this is not possible in the majority of the cases, but LSP or HSP BL Lacs can still be identified by using radio-optical-X-ray spectral indices (Padovani et al. 2003; Giommi et al. 2005). The frequency of the synchrotron peak is estimated using the broadband spectral indices between radio and optical wavelengths $\left(\alpha_{\text {ro }}\right)$ and the optical and X-ray wavelengths $\left(\alpha_{\mathrm{ox}}\right)$ and extrapolating the spectral shape of BL Lacs to infrared frequency, where the peak is expected to be located according to accepted BL Lacs SED


Figure 8. Histogram of the distributions of the CD parameter for the BZBs (black) and the BZQs (blue) (left panel), and their cumulative distribution (right panel; see Section 5 for more details).

(A color version of this figure is available in the online journal.) 




Figure 9. Scatterplot of the $\alpha_{\gamma}$ and the CD parameter for the BZBs (blue symbols) and the BZQs (red symbols).

(A color version of this figure is available in the online journal.)

model. WISE allows to directly observe the peak or a spectral interval very close to where the synchrotron emission peaks are expected.

In this paper, we adopt the SED classification criterion for the BL Lacs for the Fermi-WISE blazars sample distinguishing HSPs, ISPs, and LSPs according to the 2LAC sample (Ackermann et al. 2011). For these three families of BL Lacs we studied the relation between the two spectral indices $\alpha_{\gamma}-\alpha_{\mathrm{IR}}$. We note that, as shown in Figure 10, there is a clear distinction between the two classes in the $\alpha_{\gamma}$; however, it is more evident in the $\alpha_{\mathrm{IR}}$ distribution (a $94 \%$ level of significance has been obtained with a K-S test). A similar distinction between BL Lacs subclasses is visible in the color-magnitude plot produced using the [3.4]-[4.6] $\mu \mathrm{m}$ color and the [3.4] $\mu \mathrm{m}$ magnitude in Figure 13 in Appendix A. The three families have clearly separated color distributions, with HSPs, ISPs, and LSPs with average values of the [3.4]-[4.6] $\mu \mathrm{m}$ color $0.72,0.94$, and 1.02, respectively. Finally, we calculate the correlation coefficient between the variables $\alpha_{\gamma}$ and $\alpha_{\mathrm{IR}}$, finding $r_{s}=0.61$ ( $p$-value negligible), implying that the two variables are correlated within a high level of significance. In Figure 11, the three different BL Lacs classes in the CD versus $\alpha_{\gamma}$ plane are shown. In this case, the CD distributions of the HSPs and LSPs are similar (at a $70 \%$ level of confidence, obtained with a K-S test), while, for the ISPs and the HSPs, the null hypothesis can be rejected to an $87 \%$ level of significance.

\section{SUMMARY AND DISCUSSION}

We have presented the infrared characterization of a sample of blazars detected in the $\gamma$-ray. In order to perform our selection, we considered all the blazars in the ROMABZCAT catalog (Massaro et al. 2010) that are associated with a $\gamma$-ray source in the 2FGL (The Fermi-LAT Collaboration 2011). Then, we searched for infrared counterparts in the WISE archive adopting the same criteria described in Massaro et al. (2011a; see also Section 2 for more details). The 296 WISE counterparts of the ROMA-BZCAT-Fermi blazars constitute our sample (i.e., the Fermi-WISE blazars' sample). This more accurate characterization of a sample of blazars, as obtained by combining

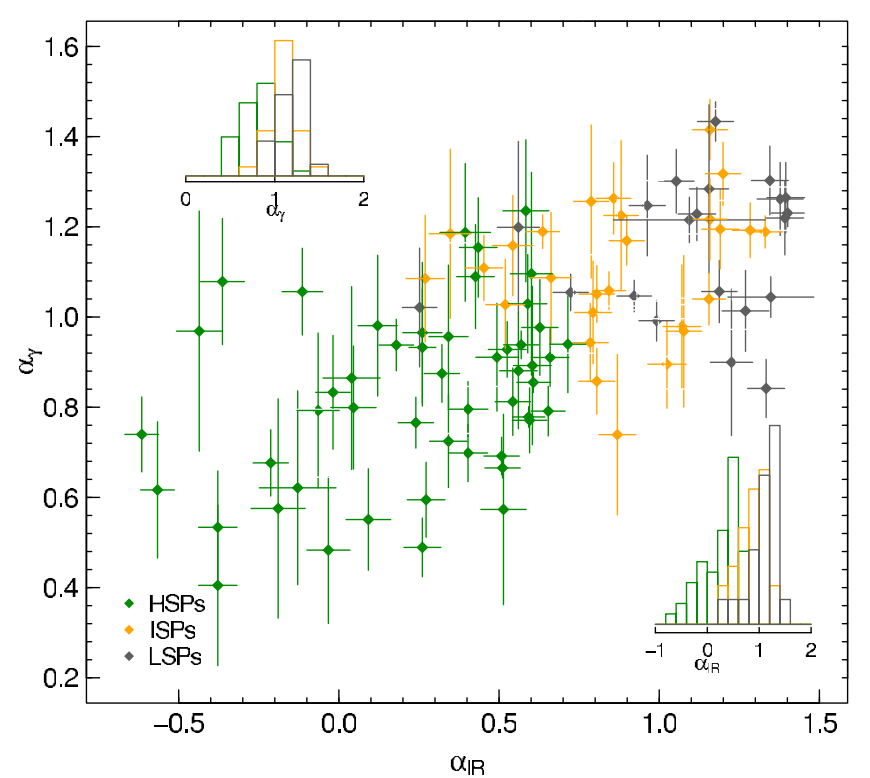

Figure 10. Scatterplot of the $\alpha_{\mathrm{IR}}-\alpha_{\gamma}$ distribution for BL Lacs. The three BL Lac subclasses of HSPs (green points), LSPs (black points), and ISPs (yellow points) are shown. We also report the histogram of the two distributions of $\alpha_{\mathrm{IR}}$ and $\alpha_{\gamma}$ that clearly show the dichotomy between the three subclasses.

(A color version of this figure is available in the online journal.)

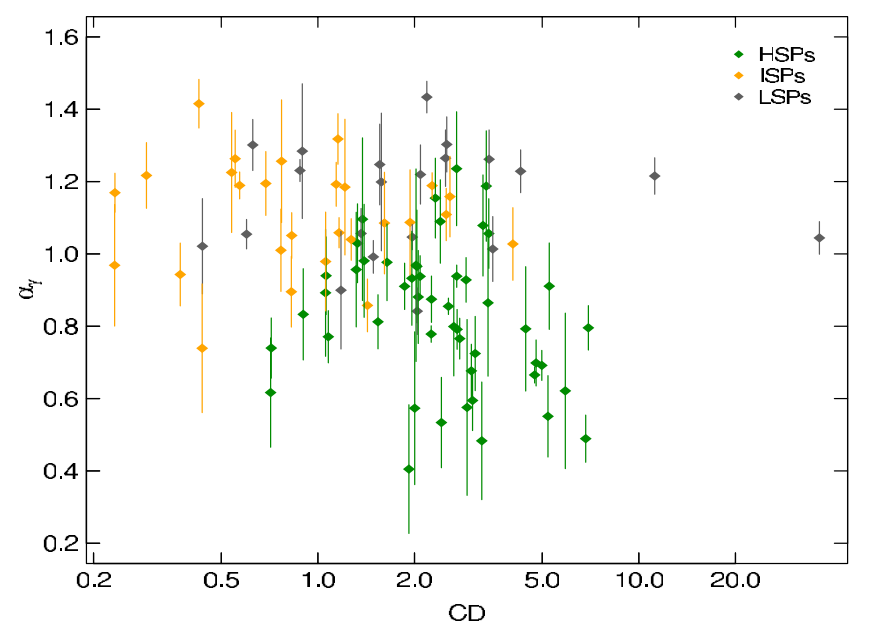

Figure 11. Scatterplot of the $\mathrm{CD}-\alpha_{\gamma}$ distribution for BL Lacs. The three BL Lac subclasses of HSPs (green points), LSPs (black points), and ISPs (yellow points) are shown (see Section 6 for more details).

(A color version of this figure is available in the online journal.)

infrared and $\gamma$-ray observations, will provide crucial clues for the understanding the unassociated Fermi objects (Massaro et al. 2011c), since we expect most of them to be blazar candidates.

We find that the Fermi-WISE blazars cover a very limited region of the [3.4]-[4.6]-[12]-[22] $\mu \mathrm{m}$ color-color plane, narrower than the similar locus found for the complete blazars population of the ROMA-BZCAT seen by WISE (i.e., the socalled WBS; Massaro et al. 2011b; see Figure 1). In particular, we show how the separation between the Fermi-WISE blazars sample and the other extragalactic sources, not dominated by synchrotron emission, is evident even with different choices of infrared colors (see Figures 3 and 12). From the three independent infrared colors obtained with WISE magnitudes, we have derived the values of the spectral indices, finding that the IR spectrum of blazars is clearly consistent with a simple power law and does not show any evidence of deviation from that. 
We investigate the properties of the relation between the spectral indices in the $\gamma$-rays and in the infrared. We found a clear trend between $\alpha_{\mathrm{IR}}-\alpha_{\gamma}$ consistent with the expectations of the SSC or the EC scenarios. In particular, in the $\alpha_{\mathrm{IR}}-\alpha_{\gamma}$ plot the dichotomy between the two main classes of blazars, BZBs and BZQs, is apparent. We also calculate the ratio between the infrared flux, integrated over the four WISE bands and the total $\gamma$-ray flux as reported in the 2FGL (The Fermi-LAT Collaboration 2011). This ratio can be used to estimate the $\mathrm{CD}$ parameter. We find that the $\mathrm{CD}$ distribution for the BZB population is more consistent with a synchrotron-dominated scenario; the BZQs, as expected, show values of CD typically higher than unity, in agreement with an inverse Compton framework and with the widely accepted EC emission model.

We also considered the BZB subclasses (i.e., HSPs, ISPs, LSPs) as defined in (Abdo et al. 2010) and we investigated their IR-to- $\gamma$-ray properties. We find a strong correlation between the spectral indices and the classification in BZB families; in particular, the HSP class appears to be very different from the LSPs in the $\alpha_{\mathrm{IR}}$ distribution.

As already shown in Massaro et al. (2011a), blazars can be separated in the WISE IR colors from other sources not dominated by synchrotron emission; however, this distinction appears to be more evident when considering those selected on the basis of their $\gamma$-ray properties. For this reason, while we are aware that the results discussed in this paper cannot be generalized, in principle, to all $\gamma$ emitting blazars because of the inhomogeneity of the parent catalog, we deem the peculiar IR features of this large sample of confirmed blazars worth investigating and interesting per se. Moreover, since blazars constitute the most detected extragalactic sources at $\gamma$-ray energies, unidentified Fermi AGN candidates or Fermi unassociated objects (The Fermi-LAT Collaboration 2011) are likely to be unknown blazars. For this reason, we are investigating the possibility of employing these new infrared correlations as diagnostic tools to associate otherwise unassociated Fermi sources with blazars, and to better categorize this interesting class of extragalactic sources (Massaro et al. 2011c).

R. D'Abrusco gratefully acknowledges the financial support of the U.S. Virtual Astronomical Observatory, which is sponsored by the National Science Foundation and the National Aeronautics and Space Administration. F. Massaro is grateful to D. Harris for constructive discussions that improved the presentation of this paper. The work at SAO is partially supported by the NASA grants NNX10AD50G and NNX10AD68G. F. Massaro also acknowledges the Fondazione Angelo Della Riccia for the grant awarded him to support his research at SAO during 2011 and the Foundation BLANCEFLOR BoncompagniLudovisi, née Bildt for the grant awarded him in 2010 to support his research. M. Ajello and F. Massaro acknowledge support from the NASA grant NNH09ZDA001N. TOPCAT ${ }^{8}$ (Taylor 2005) was used extensively in this work for the preparation and manipulation of the tabular data. Part of this work is based on archival data, software or on-line services provided by the ASI Science Data Center. This publication makes use of data products from the Wide-field Infrared Survey Explorer, which is a joint project of the University of California, Los Angeles, and the Jet Propulsion Laboratory/California Institute of Technology, funded by the National Aeronautics and Space Administration.

\footnotetext{
8 http://www.star.bris.ac.uk/ mbt/topcat/
}

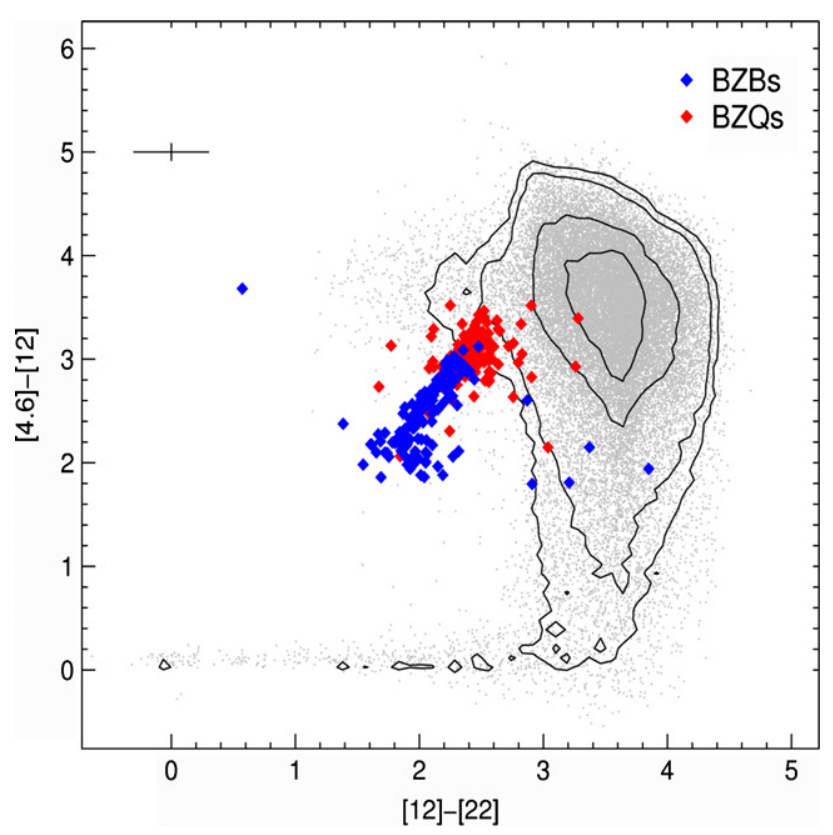

Figure 12. [4.6]-[12]-[22] $\mu \mathrm{m}$ color-color diagram of Fermi-WISE blazars sample sources. The two blazars classes of BZBs (blue) and BZQs (red) are shown. The background gray dots correspond to 453,420 WISE sources detected in a region of $56 \mathrm{deg}^{2}$ at high Galactic latitude. The isodensity curves for the WISE sources, corresponding to 50, 100, 500, and 2000 sources per unit area in the color-color plane, respectively, are shown (see Section 3). The black cross shown in the right bottom represents the typical error on the infrared colors.

(A color version of this figure is available in the online journal.)

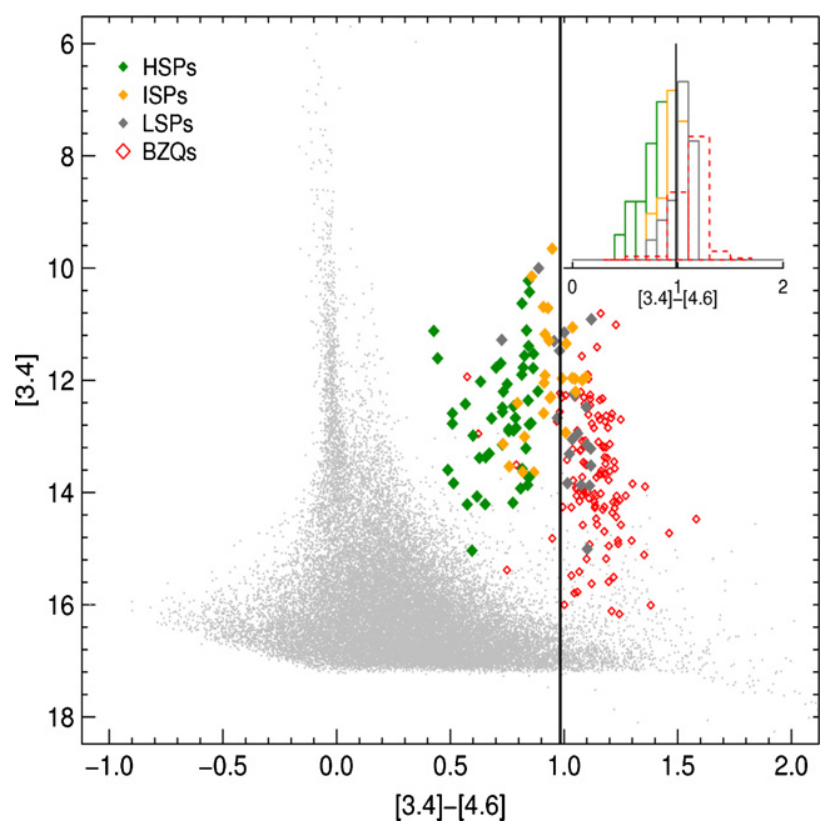

Figure 13. [3.4]-[4.6] vs. [3.4] color-magnitude diagram of the sources in the Fermi-WISE blazar sample. The BZQs (red symbols) and the HSPs, ISPs, and LSPs BL Lacs classes (see Section 6) are shown (green, orange, and gray symbols, respectively). The black vertical line corresponds to the color value associated with a power law with spectral index $\alpha_{v}=-1$.

(A color version of this figure is available in the online journal.)

\section{APPENDIX A}

\section{ADDITIONAL PLOTS}

In this Appendix, we present additional plots for the Fermi-WISE blazars sample, namely the [4.6]-[12]-[33] 

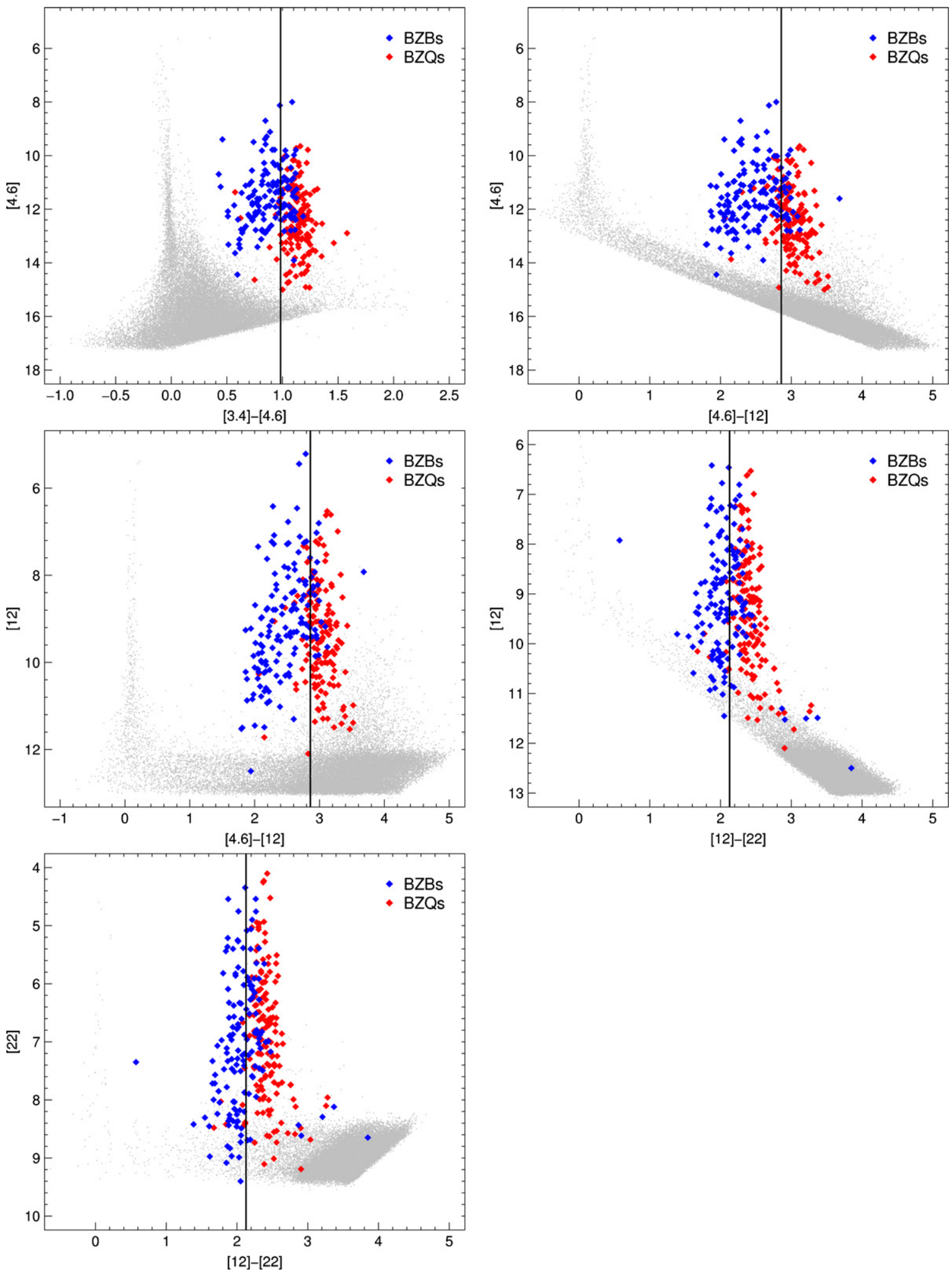

Figure 14. Color-magnitude diagrams of the Fermi-WISE sample sources. From top left: [3.4]-[4.6] vs. [4.6] plot, [4.6]-[12] vs. [4.6] plot, [4.6]-[12] vs. [12] plot, [12]-[22] vs. [12] plot, and [12]-[22] vs. [22] plot. The BZQs (red symbols) and BZBs (blue symbols) are shown in all plots. The black vertical lines correspond to the color value associated with a power law with spectral index $\alpha_{v}=-1$.

(A color version of this figure is available in the online journal.)

color-color plot (Figure 12) for BZBs and BZQs, the color-magnitude plot built using [3.4]-[4.6] color and [3.4] magnitude with the different classes of BL Lacs (Figure 13), and several color-magnitude plots with BZBs and BZQs (Figure 14) obtained using WISE photometric data.

\section{APPENDIX B}

Table 1 shows the parameters of the Fermi-WISE blazars sample. 
Table 1

Parameters of the Fermi-WISE Blazars Sample

\begin{tabular}{|c|c|c|c|c|c|c|c|}
\hline ROMA-BZCat Name & 2FGL Name & $m_{1}$ & $m_{2}$ & $m_{3}$ & $m_{4}$ & $\Gamma_{\gamma}$ & $\alpha_{\mathrm{IR}}$ \\
\hline BZBJ0035+5950 & 1ES $0033+595$ & $12.26(0.03)$ & $11.57(0.02)$ & $9.56(0.05)$ & $7.5(0.15)$ & $1.87(0.07)$ & $0.17(0.06)$ \\
\hline BZBJ0123+3420 & 1ES $0120+340$ & $13.92(0.02)$ & $13.31(0.03)$ & $11.5(0.21)$ & 8.29() & $1.53(0.21)$ & $-0.09(0.06)$ \\
\hline BZBJ0136+3905 & B3 $0133+388$ & $11.9(0.02)$ & $11.09(0.02)$ & $8.99(0.03)$ & 7.33(0.09) & $1.69(0.04)$ & $0.51(0.06)$ \\
\hline BZBJ0154+0823 & GB6 J0154+0823 & $11.91(0.02)$ & $11.0(0.02)$ & $8.52(0.03)$ & $6.57(0.07)$ & $1.86(0.07)$ & $0.81(0.06)$ \\
\hline BZBJ0154+4433 & GB6 J0154+4433 & $13.25(0.03)$ & $12.31(0.03)$ & $9.98(0.05)$ & $8.03(0.2)$ & $2.01(0.26)$ & $0.87(0.06)$ \\
\hline BZBJ0159+1047 & RX J0159.5+1047 & $12.85(0.02)$ & $12.06(0.02)$ & $9.96(0.06)$ & $8.24(0.3)$ & $2.15(0.11)$ & $0.44(0.06)$ \\
\hline BZBJ0203+7232 & S5 $0159+723$ & $11.28(0.02)$ & $10.55(0.02)$ & $8.04(0.02)$ & $5.89(0.04)$ & $2.02(0.13)$ & $0.25(0.05)$ \\
\hline BZBJ0212+2244 & MG3 J021252+2246 & $12.36(0.02)$ & $11.52(0.02)$ & $9.29(0.05)$ & $7.47(0.21)$ & $2.03(0.11)$ & $0.59(0.06)$ \\
\hline BZBJ0217+0837 & ZS 0214+083 & $10.69(0.02)$ & $9.78(0.02)$ & $7.26(0.02)$ & $5.25(0.03)$ & $1.94(0.09)$ & $0.78(0.06)$ \\
\hline BZBJ0222+4302 & $3 \mathrm{C} 66 \mathrm{~A}$ & $9.55(0.02)$ & $8.7(0.02)$ & $6.42(0.02)$ & $4.54(0.03)$ & $1.85(0.02)$ & $0.6(0.05)$ \\
\hline BZBJ0238+1636 & AO $0235+164$ & $11.78(0.02)$ & $10.66(0.02)$ & $7.7(0.02)$ & $5.39(0.03)$ & $2.02(0.02)$ & $1.39(0.05)$ \\
\hline BZBJ0243+7120 & S5 $0238+711$ & $12.95(0.03)$ & $11.89(0.02)$ & $9.33(0.03)$ & $7.03(0.09)$ & $1.9(0.16)$ & $1.23(0.07)$ \\
\hline BZBJ0258+2030 & MG3 J025805+2029 & $12.84(0.03)$ & $11.82(0.02)$ & $9.48(0.05)$ & $7.52(0.22)$ & $2.19(0.17)$ & $1.1(0.06)$ \\
\hline BZBJ0303-2407 & PKS 0301-243 & $11.11(0.02)$ & $10.28(0.02)$ & $7.96(0.02)$ & $6.09(0.05)$ & $1.94(0.03)$ & $0.57(0.06)$ \\
\hline BZBJ0303+4716 & $4 C+47.08$ & $11.08(0.02)$ & $9.98(0.02)$ & $7.02(0.02)$ & $4.76(0.02)$ & $2.24(0.07)$ & $1.35(0.05)$ \\
\hline BZBJ0304-2832 & RBS 0385 & $15.04(0.04)$ & $14.44(0.06)$ & 12.5() & 8.65() & $1.62(0.21)$ & $-0.13(0.12)$ \\
\hline BZBJ0316-2607 & RBS 0405 & $12.99(0.03)$ & $12.24(0.02)$ & $10.06(0.05)$ & $8.45(0.24)$ & $1.87(0.14)$ & $0.33(0.06)$ \\
\hline BZBJ0316+0904 & GB6 J0316+0904 & $11.56(0.02)$ & $10.74(0.02)$ & $8.47(0.03)$ & $6.58(0.07)$ & $1.81(0.07)$ & $0.54(0.06)$ \\
\hline BZBJ0319+1845 & RBS 0413 & $13.31(0.03)$ & $12.64(0.03)$ & $10.55(0.09)$ & 8.49() & $1.55(0.11)$ & $0.09(0.07)$ \\
\hline BZBJ0321+2326 & MG3 J032201+2336 & $12.67(0.03)$ & $11.88(0.02)$ & $9.77(0.05)$ & $7.46(0.13)$ & $2.09(0.12)$ & $0.43(0.06)$ \\
\hline BZBJ0325-1646 & RBS 0421 & $13.15(0.02)$ & $12.42(0.03)$ & $10.37(0.06)$ & $8.38(0.27)$ & $1.97(0.16)$ & $0.26(0.06)$ \\
\hline BZBJ0326+0225 & $1 \mathrm{H} 0323+022$ & $12.98(0.03)$ & $12.38(0.03)$ & $10.24(0.07)$ & $8.36(0.28)$ & $2.06(0.1)$ & $-0.11(0.06)$ \\
\hline BZBJ0334-4008 & PKS 0332-403 & $11.94(0.02)$ & $10.84(0.02)$ & $7.92(0.02)$ & $5.65(0.03)$ & $2.19(0.04)$ & $1.33(0.06)$ \\
\hline BZBJ0334-3725 & PMN J0334-3725 & $11.47(0.02)$ & $10.49(0.02)$ & $7.87(0.02)$ & $5.78(0.04)$ & $1.99(0.05)$ & $0.99(0.06)$ \\
\hline BZBJ0340-2119 & PKS 0338-214 & $11.99(0.02)$ & $11.0(0.02)$ & $8.22(0.02)$ & $6.03(0.04)$ & $2.43(0.14)$ & $1.02(0.06)$ \\
\hline BZBJ0357-4955 & PKS 0355-500 & $12.32(0.02)$ & $11.38(0.02)$ & $8.81(0.02)$ & $6.87(0.06)$ & $1.74(0.18)$ & $0.87(0.06)$ \\
\hline BZBJ0424+0036 & PKS 0422+00 & $11.15(0.02)$ & $10.15(0.02)$ & $7.47(0.02)$ & $5.38(0.03)$ & $2.3(0.07)$ & $1.05(0.06)$ \\
\hline BZBJ0428-3756 & PKS 0426-380 & $10.84(0.02)$ & $9.81(0.02)$ & $7.11(0.02)$ & $4.9(0.03)$ & $1.95(0.02)$ & $1.13(0.05)$ \\
\hline BZBJ0430-2507 & PMN J0430-2507 & $13.69(0.03)$ & $12.85(0.03)$ & $10.32(0.06)$ & $8.44(0.27)$ & $2.2(0.19)$ & $0.56(0.07)$ \\
\hline BZBJ0433+2905 & MG2 J043337+2905 & $15.01(0.06)$ & $13.9(0.06)$ & $11.3(0.18)$ & 8.43() & $2.04(0.05)$ & $1.35(0.13)$ \\
\hline BZBJ0434-2015 & TXS 0431-203 & $13.39(0.03)$ & $12.41(0.03)$ & $9.8(0.04)$ & $7.59(0.14)$ & $2.22(0.13)$ & $1.01(0.06)$ \\
\hline BZBJ0448-1632 & RBS 0589 & $13.93(0.03)$ & $13.12(0.03)$ & $10.89(0.08)$ & $8.97(0.33)$ & $1.91(0.12)$ & $0.49(0.07)$ \\
\hline BZBJ0449-4350 & PKS 0447-439 & $10.43(0.02)$ & $9.58(0.02)$ & $7.28(0.02)$ & $5.44(0.03)$ & $1.86(0.02)$ & $0.61(0.05)$ \\
\hline BZBJ0507+6737 & 1ES $0502+675$ & $12.56(0.03)$ & $11.83(0.02)$ & $9.85(0.04)$ & $8.3(0.23)$ & $1.49(0.07)$ & $0.26(0.06)$ \\
\hline BZBJ0509+0541 & TXS 0506+056 & $10.71(0.02)$ & $9.78(0.02)$ & $7.28(0.02)$ & $5.27(0.03)$ & $2.06(0.04)$ & $0.84(0.05)$ \\
\hline BZBJ0536-3343 & 1RXS J053629.4-334302 & $12.98(0.02)$ & $12.28(0.02)$ & $10.22(0.05)$ & $7.95(0.15)$ & $2.39(0.06)$ & $0.19(0.06)$ \\
\hline BZBJ0538-4405 & PKS 0537-441 & $9.09(0.02)$ & $8.0(0.02)$ & $5.21(0.02)$ & $3.04(0.02)$ & $2.01(0.02)$ & $1.3(0.05)$ \\
\hline BZBJ0558-7459 & PKS 0600-749 & $12.25(0.02)$ & $11.28(0.02)$ & $8.67(0.02)$ & $6.6(0.05)$ & $2.09(0.14)$ & $0.98(0.06)$ \\
\hline BZBJ0607+4739 & TXS 0603+476 & $11.18(0.02)$ & $10.26(0.02)$ & $7.8(0.02)$ & $5.83(0.04)$ & $2.05(0.06)$ & $0.81(0.06)$ \\
\hline BZBJ0612+4122 & B3 $0609+413$ & $11.82(0.03)$ & $10.81(0.02)$ & $8.12(0.02)$ & $6.02(0.04)$ & $2.03(0.05)$ & $1.09(0.06)$ \\
\hline BZBJ0617+5701 & 87 GB $061258.1+570222$ & $11.97(0.03)$ & $10.98(0.02)$ & $8.36(0.02)$ & $6.33(0.05)$ & $1.9(0.1)$ & $1.02(0.06)$ \\
\hline BZBJ0625+4440 & GB6 J0625+4440 & $12.92(0.03)$ & $11.94(0.02)$ & $9.3(0.03)$ & $7.29(0.1)$ & $1.91(0.14)$ & $1.0(0.06)$ \\
\hline BZBJ0629-1959 & PKS 0627-199 & $12.38(0.03)$ & $11.26(0.02)$ & $8.41(0.02)$ & $6.16(0.04)$ & $2.19(0.06)$ & $1.42(0.06)$ \\
\hline BZBJ0650+2503 & $1 \mathrm{ES} 0647+250$ & $12.2(0.03)$ & $11.47(0.02)$ & $9.37(0.04)$ & $7.72(0.17)$ & $1.59(0.08)$ & $0.27(0.06)$ \\
\hline BZBJ0710+5908 & $1 \mathrm{H} 0658+595$ & $12.59(0.02)$ & $12.08(0.02)$ & $10.22(0.06)$ & $8.18(0.19)$ & $1.53(0.12)$ & $-0.38(0.06)$ \\
\hline BZBJ0712+5033 & GB6 J0712+5033 & $12.27(0.02)$ & $11.22(0.02)$ & $8.46(0.02)$ & $6.27(0.06)$ & $2.06(0.07)$ & $1.19(0.06)$ \\
\hline BZBJ0721+7120 & S5 $0716+71$ & $9.1(0.02)$ & $8.13(0.02)$ & $5.44(0.02)$ & $3.4(0.02)$ & $2.01(0.02)$ & $0.98(0.05)$ \\
\hline BZBJ0730+3307 & 1RXS J073026.0+330727 & $12.79(0.03)$ & $11.94(0.02)$ & $9.67(0.05)$ & $8.0(0.2)$ & $1.89(0.18)$ & $0.6(0.06)$ \\
\hline BZBJ0738+1742 & PKS 0735+17 & $11.3(0.02)$ & $10.34(0.02)$ & $7.74(0.02)$ & $5.72(0.04)$ & $2.05(0.03)$ & $0.92(0.05)$ \\
\hline BZBJ0744+7433 & MS 0737.9+7441 & $13.37(0.03)$ & $12.72(0.03)$ & $10.74(0.09)$ & 8.83() & $1.8(0.14)$ & $0.05(0.07)$ \\
\hline BZBJ0753+5352 & $4 \mathrm{C}+54.15$ & $13.87(0.03)$ & $12.79(0.03)$ & $9.86(0.05)$ & $7.49(0.13)$ & $2.01(0.09)$ & $1.27(0.07)$ \\
\hline BZBJ0754+4823 & GB1 $0751+485$ & $11.97(0.03)$ & $10.92(0.02)$ & $8.15(0.02)$ & $5.91(0.04)$ & $2.19(0.09)$ & $1.19(0.06)$ \\
\hline BZBJ0757+0956 & PKS 0754+100 & $12.0(0.02)$ & $10.92(0.02)$ & $8.04(0.02)$ & $5.66(0.04)$ & $2.19(0.06)$ & $1.28(0.06)$ \\
\hline BZBJ0805+7534 & RX J0805.4+7534 & $12.42(0.02)$ & $11.86(0.02)$ & $9.8(0.04)$ & $8.04(0.16)$ & $1.68(0.07)$ & $-0.21(0.06)$ \\
\hline BZBJ0809+5218 & 1ES $0806+524$ & $11.78(0.02)$ & $11.07(0.02)$ & $8.96(0.03)$ & $7.11(0.11)$ & $1.94(0.06)$ & $0.18(0.05)$ \\
\hline BZBJ0811+0146 & OJ 014 & $13.88(0.03)$ & $12.77(0.03)$ & $9.65(0.05)$ & $7.17(0.1)$ & $2.26(0.08)$ & $1.38(0.07)$ \\
\hline BZBJ0814+6431 & GB6 J0814+6431 & $11.29(0.02)$ & $10.36(0.02)$ & $7.84(0.02)$ & $5.86(0.04)$ & $2.26(0.08)$ & $0.86(0.05)$ \\
\hline BZBJ0816-1311 & PMN J0816-1311 & $12.9(0.03)$ & $12.12(0.02)$ & $10.12(0.05)$ & $8.15(0.21)$ & $1.8(0.06)$ & $0.4(0.06)$ \\
\hline BZBJ0816+5739 & SBS $0812+578$ & $12.76(0.02)$ & $11.91(0.02)$ & $9.51(0.04)$ & $7.41(0.1)$ & $1.98(0.11)$ & $0.63(0.06)$ \\
\hline BZBJ0817+3243 & RX J0817.9+3243 & $14.18(0.03)$ & $13.41(0.04)$ & $11.02(0.12)$ & $8.99(0.44)$ & $2.19(0.15)$ & $0.39(0.08)$ \\
\hline BZBJ0818+4222 & S4 0814+42 & $12.7(0.03)$ & $11.58(0.02)$ & $8.59(0.03)$ & $6.27(0.05)$ & $2.14(0.03)$ & $1.41(0.06)$ \\
\hline BZBJ0817-0933 & TXS 0815-094 & $12.43(0.03)$ & $11.42(0.02)$ & $8.78(0.03)$ & $6.51(0.06)$ & $2.04(0.09)$ & $1.06(0.06)$ \\
\hline BZBJ0819+2747 & 5C 07.119 & $13.3(0.03)$ & $12.24(0.02)$ & $9.43(0.04)$ & $6.99(0.08)$ & $2.26(0.22)$ & $1.23(0.06)$ \\
\hline BZBJ0819-0756 & RX J0819.2-0756 & $14.21(0.03)$ & $13.64(0.04)$ & $11.49(0.16)$ & $8.12(0.26)$ & $1.58(0.24)$ & $-0.19(0.08)$ \\
\hline BZBJ0834+4403 & B3 $0831+442$ & $12.85(0.03)$ & $11.91(0.02)$ & $9.31(0.04)$ & $7.2(0.09)$ & $2.04(0.19)$ & $0.87(0.06)$ \\
\hline BZBJ0839+1802 & TXS $0836+182$ & $12.76(0.03)$ & $11.88(0.02)$ & $9.39(0.04)$ & $7.39(0.12)$ & $2.46(0.2)$ & $0.72(0.06)$ \\
\hline
\end{tabular}


Table 1

(Continued)

\begin{tabular}{|c|c|c|c|c|c|c|c|}
\hline ROMA-BZCat Name & 2FGL Name & $m_{1}$ & $m_{2}$ & $m_{3}$ & $m_{4}$ & $\Gamma_{\gamma}$ & $\alpha_{\mathrm{IR}}$ \\
\hline BZBJ0839+3540 & FIRST J083943.3+354001 & $13.54(0.03)$ & $12.64(0.03)$ & $10.31(0.08)$ & $8.36(0.33)$ & $1.92(0.18)$ & $0.73(0.07)$ \\
\hline BZBJ0844+5312 & BZB J0844+5312 & $13.64(0.03)$ & $12.77(0.03)$ & $10.25(0.06)$ & $8.25(0.3)$ & $2.09(0.15)$ & $0.66(0.06)$ \\
\hline BZBJ0847+1133 & RX J0847.1+1133 & $13.38(0.03)$ & $12.76(0.03)$ & $10.87(0.12)$ & $8.68(0.42)$ & $1.48(0.16)$ & $-0.03(0.07)$ \\
\hline BZBJ0856+2057 & PMN J0856-1105 & $13.5(0.03)$ & $12.6(0.03)$ & $10.06(0.08)$ & 7.9() & $2.14(0.14)$ & $0.76(0.07)$ \\
\hline BZBJ0902+2050 & NVSS J090226+205045 & $12.05(0.03)$ & $11.13(0.02)$ & $8.71(0.03)$ & $6.7(0.07)$ & $2.01(0.11)$ & $0.79(0.06)$ \\
\hline BZBJ0910+3329 & Ton 1015 & $12.2(0.02)$ & $11.31(0.02)$ & $8.89(0.03)$ & $6.97(0.08)$ & $1.94(0.11)$ & $0.71(0.06)$ \\
\hline BZBJ0915+2933 & B2 0912+29 & $12.07(0.02)$ & $11.32(0.02)$ & $9.05(0.03)$ & $7.19(0.1)$ & $1.87(0.06)$ & $0.32(0.06)$ \\
\hline BZBJ0929+5013 & GB6 J0929+5013 & $12.93(0.03)$ & $11.93(0.02)$ & $9.13(0.03)$ & $6.85(0.06)$ & $1.98(0.14)$ & $1.07(0.06)$ \\
\hline BZBJ0929+8612 & S5 0916+864 & $12.86(0.02)$ & $11.84(0.02)$ & $9.09(0.03)$ & $6.84(0.06)$ & $2.05(0.09)$ & $1.13(0.06)$ \\
\hline BZBJ0940+6148 & RX J0940.3+6148 & $13.83(0.03)$ & $13.32(0.03)$ & $11.52(0.14)$ & 8.62() & $2.08(0.14)$ & $-0.36(0.07)$ \\
\hline BZBJ0945+5757 & GB6 J0945+5757 & $12.52(0.02)$ & $11.75(0.02)$ & $9.39(0.03)$ & $7.46(0.08)$ & $2.16(0.14)$ & $0.37(0.06)$ \\
\hline BZBJ0958+6533 & S4 0954+65 & $11.06(0.02)$ & $10.02(0.02)$ & $7.22(0.02)$ & $5.08(0.03)$ & $2.42(0.07)$ & $1.16(0.06)$ \\
\hline BZBJ1018+5911 & TXS $1015+594$ & $13.54(0.03)$ & $12.78(0.03)$ & $10.3(0.06)$ & $8.2(0.21)$ & $2.18(0.19)$ & $0.35(0.07)$ \\
\hline BZBJ1019+6320 & GB6 J1019+6319 & $12.82(0.02)$ & $11.81(0.02)$ & $9.1(0.03)$ & $6.96(0.06)$ & $2.18(0.19)$ & $1.09(0.06)$ \\
\hline BZBJ1058-8003 & PKS 1057-79 & $11.52(0.02)$ & $10.45(0.02)$ & $7.6(0.02)$ & $5.4(0.03)$ & $2.05(0.09)$ & $1.27(0.05)$ \\
\hline BZBJ1110+7133 & $87 \mathrm{~GB} 110723.4+715023$ & $13.73(0.03)$ & $12.89(0.03)$ & $10.67(0.07)$ & $8.61(0.26)$ & $2.1(0.22)$ & $0.6(0.07)$ \\
\hline BZBJ1136+7009 & Mkn 180 & $11.12(0.02)$ & $10.69(0.02)$ & $8.69(0.02)$ & $6.75(0.06)$ & $1.74(0.08)$ & $-0.62(0.05)$ \\
\hline BZBJ1223+8040 & S5 $1221+80$ & $13.52(0.03)$ & $12.4(0.02)$ & $9.44(0.03)$ & $7.11(0.07)$ & $2.26(0.08)$ & $1.4(0.06)$ \\
\hline BZBJ1312-2156 & PKS 1309-216 & $12.06(0.03)$ & $11.12(0.02)$ & $8.79(0.03)$ & $6.9(0.1)$ & $2.02(0.07)$ & $0.9(0.06)$ \\
\hline BZBJ1352-4412 & PKS 1349-439 & $12.6(0.03)$ & $11.51(0.02)$ & $8.69(0.03)$ & $6.52(0.07)$ & $2.13(0.17)$ & $1.32(0.06)$ \\
\hline BZBJ1357+0128 & RX J1357.6+0128 & $13.68(0.03)$ & $12.85(0.03)$ & $10.45(0.08)$ & $8.46(0.37)$ & $2.28(0.16)$ & $0.56(0.07)$ \\
\hline BZBJ1359-3746 & PMN J1359-3746 & $12.74(0.03)$ & $11.86(0.02)$ & $9.31(0.03)$ & $7.23(0.1)$ & $1.63(0.17)$ & $0.7(0.06)$ \\
\hline BZBJ1418-0233 & BZB J1418-0233 & $11.95(0.02)$ & $11.08(0.02)$ & $8.79(0.03)$ & $7.07(0.1)$ & $1.7(0.07)$ & $0.68(0.06)$ \\
\hline BZBJ1427+2348 & PKS $1424+240$ & $10.22(0.02)$ & $9.38(0.02)$ & $7.08(0.02)$ & $5.21(0.03)$ & $1.78(0.02)$ & $0.59(0.05)$ \\
\hline BZBJ1439-1531 & PKS $1437-153$ & $13.41(0.03)$ & $12.33(0.03)$ & $9.38(0.04)$ & $7.17(0.12)$ & $2.4(0.16)$ & $1.28(0.07)$ \\
\hline BZBJ1440+0610 & PMN J1440+0610 & $13.01(0.03)$ & $12.18(0.03)$ & $9.81(0.05)$ & $8.42(0.3)$ & $2.16(0.11)$ & $0.54(0.06)$ \\
\hline BZBJ1442+1200 & 1ES $1440+122$ & $12.77(0.02)$ & $12.26(0.02)$ & $10.38(0.07)$ & 8.37() & $1.41(0.18)$ & $-0.38(0.06)$ \\
\hline BZBJ1443-3908 & PKS 1440-389 & $11.7(0.02)$ & $10.97(0.02)$ & $8.79(0.03)$ & $6.89(0.08)$ & $1.77(0.06)$ & $0.24(0.06)$ \\
\hline BZBJ1501+2238 & MS $1458.8+2249$ & $11.39(0.02)$ & $10.55(0.02)$ & $8.21(0.02)$ & $6.33(0.05)$ & $1.77(0.07)$ & $0.6(0.05)$ \\
\hline BZBJ1503-1541 & RBS 1457 & $13.74(0.03)$ & $13.12(0.03)$ & $10.93(0.13)$ & $9.08(0.54)$ & $1.8(0.15)$ & $-0.08(0.07)$ \\
\hline BZBJ1506+0814 & PMN J1506+0814 & $12.9(0.03)$ & $12.15(0.02)$ & $9.98(0.05)$ & $7.87(0.16)$ & $1.96(0.16)$ & $0.34(0.06)$ \\
\hline BZBJ1516+1932 & PKS 1514+197 & $12.26(0.02)$ & $11.2(0.02)$ & $8.3(0.02)$ & $6.09(0.05)$ & $2.46(0.16)$ & $1.23(0.06)$ \\
\hline BZBJ1517-2422 & AP Librae & $10.0(0.02)$ & $9.11(0.02)$ & $6.46(0.02)$ & $4.34(0.02)$ & $2.05(0.04)$ & $0.72(0.06)$ \\
\hline BZBJ $1522-2730$ & PKS 1519-273 & $13.84(0.12)$ & $12.82(0.08)$ & $9.83(0.1)$ & $7.61(0.36)$ & $2.22(0.05)$ & $1.09(0.24)$ \\
\hline BZBJ1534+3715 & RGB J1534+372 & $13.45(0.03)$ & $12.81(0.03)$ & $10.84(0.09)$ & $8.7(0.37)$ & $2.15(0.16)$ & $0.0(0.07)$ \\
\hline BZBJ1540+8155 & 1ES $1544+820$ & $12.9(0.02)$ & $12.2(0.02)$ & $10.15(0.04)$ & $8.26(0.17)$ & $1.48(0.16)$ & $0.16(0.06)$ \\
\hline BZBJ1546+0819 & 1RXS J154604.6+081912 & $13.58(0.03)$ & $12.76(0.03)$ & $10.59(0.08)$ & $8.97(0.42)$ & $1.57(0.21)$ & $0.51(0.07)$ \\
\hline BZBJ1548-2251 & PMN J1548-2251 & $12.49(0.02)$ & $11.76(0.02)$ & $9.64(0.04)$ & $7.78(0.15)$ & $1.93(0.13)$ & $0.26(0.04)$ \\
\hline BZBJ $1552+0850$ & TXS 1549+089 & $12.28(0.03)$ & $11.29(0.02)$ & $8.57(0.03)$ & $6.44(0.06)$ & $2.0(0.16)$ & $1.02(0.06)$ \\
\hline BZBJ1555+1111 & PG $1553+113$ & $10.63(0.02)$ & $9.82(0.02)$ & $7.62(0.02)$ & $5.82(0.04)$ & $1.67(0.02)$ & $0.51(0.06)$ \\
\hline BZBJ $1607+1551$ & $4 C+15.54$ & $13.31(0.02)$ & $12.29(0.02)$ & $9.41(0.04)$ & $7.0(0.08)$ & $2.23(0.06)$ & $1.12(0.06)$ \\
\hline BZBJ1610-6649 & PMN J1610-6649 & $12.46(0.03)$ & $11.69(0.02)$ & $9.59(0.04)$ & $7.85(0.15)$ & $1.7(0.06)$ & $0.4(0.06)$ \\
\hline BZBJ1630+5221 & TXS $1629+524$ & $13.63(0.03)$ & $12.81(0.02)$ & $10.66(0.06)$ & $8.8(0.26)$ & $2.03(0.1)$ & $0.52(0.06)$ \\
\hline BZBJ1642-0621 & TXS 1639-062 & $13.45(0.03)$ & $12.26(0.02)$ & $9.18(0.03)$ & $6.82(0.08)$ & $2.37(0.13)$ & $1.59(0.06)$ \\
\hline BZBJ1653+3945 & Mkn 501 & $9.86(0.02)$ & $9.4(0.02)$ & $7.34(0.02)$ & $5.4(0.03)$ & $1.74(0.03)$ & $-0.52(0.05)$ \\
\hline BZBJ1719+1745 & PKS $1717+177$ & $12.48(0.03)$ & $11.38(0.02)$ & $8.46(0.02)$ & $6.24(0.04)$ & $1.84(0.06)$ & $1.33(0.06)$ \\
\hline BZBJ1725+1152 & $1 \mathrm{H} 1720+117$ & $11.77(0.02)$ & $10.95(0.02)$ & $8.76(0.03)$ & $6.97(0.08)$ & $1.93(0.06)$ & $0.53(0.06)$ \\
\hline BZBJ $1725+5851$ & $7 \mathrm{C} 1724+5854$ & $12.59(0.02)$ & $11.68(0.02)$ & $9.2(0.03)$ & $7.33(0.08)$ & $2.26(0.17)$ & $0.79(0.06)$ \\
\hline BZBJ1728+5013 & I Zw 187 & $12.02(0.02)$ & $11.39(0.02)$ & $9.17(0.02)$ & $7.15(0.07)$ & $1.83(0.13)$ & $-0.02(0.05)$ \\
\hline BZBJ1730+3714 & GB6 J1730+3714 & $13.14(0.02)$ & $12.41(0.02)$ & $10.17(0.04)$ & $8.18(0.18)$ & $2.09(0.14)$ & $0.27(0.06)$ \\
\hline BZBJ1739+4737 & S4 $1738+47$ & $13.26(0.02)$ & $12.18(0.02)$ & $9.25(0.03)$ & $6.94(0.06)$ & $2.09(0.15)$ & $1.26(0.06)$ \\
\hline BZBJ1742+5945 & RGB $1742+597$ & $12.3(0.02)$ & $11.36(0.02)$ & $8.78(0.02)$ & $6.78(0.05)$ & $2.23(0.17)$ & $0.88(0.05)$ \\
\hline BZBJ1743+1935 & S3 $1741+19$ & $11.61(0.02)$ & $11.16(0.02)$ & $9.22(0.03)$ & $7.3(0.1)$ & $1.62(0.15)$ & $-0.57(0.05)$ \\
\hline BZBJ $1748+7005$ & S4 $1749+70$ & $11.96(0.02)$ & $10.92(0.02)$ & $8.14(0.02)$ & $5.96(0.03)$ & $2.04(0.06)$ & $1.16(0.05)$ \\
\hline BZBJ1749+4321 & B3 $1747+433$ & $13.22(0.03)$ & $12.1(0.02)$ & $9.07(0.03)$ & $6.8(0.06)$ & $2.22(0.08)$ & $1.39(0.06)$ \\
\hline BZBJ1756+5522 & 1RXS J175615.5+552217 & $14.07(0.03)$ & $13.45(0.03)$ & $11.45(0.09)$ & $9.4(0.48)$ & $1.79(0.17)$ & $-0.06(0.07)$ \\
\hline BZBJ $1800+7828$ & S5 $1803+784$ & $10.91(0.02)$ & $9.79(0.02)$ & $6.8(0.02)$ & $4.54(0.02)$ & $2.23(0.03)$ & $1.4(0.05)$ \\
\hline BZBJ1806+6949 & $3 \mathrm{C} 371$ & $10.15(0.02)$ & $9.29(0.02)$ & $6.77(0.02)$ & $4.75(0.02)$ & $2.19(0.04)$ & $0.64(0.05)$ \\
\hline BZBJ1809+2910 & MG2 J180948+2910 & $12.33(0.02)$ & $11.37(0.02)$ & $8.77(0.03)$ & $6.74(0.06)$ & $2.04(0.11)$ & $0.95(0.06)$ \\
\hline BZBJ1813+3144 & B2 $1811+31$ & $12.4(0.02)$ & $11.61(0.02)$ & $9.4(0.04)$ & $7.72(0.15)$ & $2.11(0.07)$ & $0.45(0.06)$ \\
\hline BZBJ1813+0615 & TXS $1811+062$ & $12.98(0.03)$ & $11.91(0.02)$ & $9.12(0.03)$ & $6.84(0.07)$ & $1.97(0.13)$ & $1.24(0.07)$ \\
\hline BZBJ $1824+5651$ & $4 C+56.27$ & $12.24(0.02)$ & $11.19(0.02)$ & $8.22(0.02)$ & $5.92(0.04)$ & $2.43(0.04)$ & $1.18(0.06)$ \\
\hline BZBJ1829+5402 & 1RXS J182925.7+540255 & $13.21(0.02)$ & $12.38(0.02)$ & $10.21(0.04)$ & $8.32(0.14)$ & $1.88(0.13)$ & $0.56(0.06)$ \\
\hline BZBJ1832-5659 & PMN J1832-5659 & $13.11(0.03)$ & $12.14(0.02)$ & $9.39(0.04)$ & $7.23(0.09)$ & $2.3(0.13)$ & $0.95(0.06)$ \\
\hline BZBJ1838+4802 & GB6 J1838+4802 & $12.87(0.03)$ & $12.11(0.02)$ & $10.0(0.04)$ & $8.04(0.12)$ & $1.72(0.1)$ & $0.34(0.06)$ \\
\hline BZBJ1849-4314 & PMN J1849-4314 & $11.93(0.03)$ & $10.97(0.02)$ & $8.4(0.02)$ & $6.34(0.05)$ & $2.02(0.09)$ & $0.93(0.06)$ \\
\hline
\end{tabular}


Table 1

(Continued)

\begin{tabular}{|c|c|c|c|c|c|c|c|}
\hline ROMA-BZCat Name & 2FGL Name & $m_{1}$ & $m_{2}$ & $m_{3}$ & $m_{4}$ & $\Gamma_{\gamma}$ & $\alpha_{\mathrm{IR}}$ \\
\hline BZBJ1917-1921 & 1H 1914-194 & $11.53(0.03)$ & $10.66(0.02)$ & $8.33(0.02)$ & $6.38(0.06)$ & $1.91(0.06)$ & $0.66(0.06)$ \\
\hline BZBJ1918-4111 & PMN J1918-4111 & $12.95(0.03)$ & $11.96(0.02)$ & $9.41(0.04)$ & $7.32(0.09)$ & $1.84(0.06)$ & $1.02(0.06)$ \\
\hline BZBJ1921-1607 & PMN J1921-1607 & $12.38(0.03)$ & $11.6(0.02)$ & $7.92(0.06)$ & $7.35(0.24)$ & $1.74(0.1)$ & $0.39(0.06)$ \\
\hline BZBJ1931+0937 & RX J1931.1+0937 & $11.79(0.04)$ & $11.12(0.03)$ & $9.26(0.04)$ & $7.57(0.12)$ & $2.36(0.07)$ & $0.09(0.08)$ \\
\hline BZBJ1936-4719 & PMN J1936-4719 & $13.52(0.03)$ & $12.88(0.03)$ & $10.78(0.09)$ & $8.73(0.37)$ & $1.64(0.16)$ & $-0.0(0.07)$ \\
\hline BZBJ1945-3111 & PKS 1942-313 & $13.41(0.04)$ & $12.3(0.03)$ & $9.68(0.05)$ & $7.42(0.15)$ & $2.29(0.15)$ & $1.36(0.08)$ \\
\hline BZBJ2005+7752 & S5 $2007+77$ & $11.06(0.02)$ & $10.02(0.02)$ & $7.26(0.02)$ & $5.06(0.02)$ & $2.22(0.09)$ & $1.16(0.05)$ \\
\hline BZBJ2009-4849 & PKS 2005-489 & $10.23(0.02)$ & $9.49(0.02)$ & $7.23(0.02)$ & $5.36(0.03)$ & $1.78(0.05)$ & $0.29(0.05)$ \\
\hline BZBJ2009+7229 & $4 \mathrm{C}+72.28$ & $13.16(0.02)$ & $12.06(0.02)$ & $9.21(0.03)$ & $6.88(0.05)$ & $2.3(0.08)$ & $1.35(0.06)$ \\
\hline BZBJ2015-0137 & PKS 2012-017 & $12.67(0.02)$ & $11.7(0.02)$ & $8.98(0.03)$ & $6.88(0.07)$ & $2.25(0.11)$ & $0.96(0.06)$ \\
\hline BZBJ2022+7611 & S5 $2023+760$ & $12.2(0.02)$ & $11.15(0.02)$ & $8.25(0.02)$ & $6.04(0.03)$ & $2.32(0.07)$ & $1.2(0.06)$ \\
\hline BZQJ0044-8422 & PKS 0044-84 & $13.73(0.03)$ & $12.65(0.03)$ & $9.81(0.03)$ & 7.44(0.09) & $2.53(0.11)$ & $1.28(0.06)$ \\
\hline BZQJ0102+4214 & CRATES J0102+4214 & $14.58(0.04)$ & $13.5(0.04)$ & $10.45(0.07)$ & $8.0(0.18)$ & $2.61(0.09)$ & $1.29(0.08)$ \\
\hline BZQJ0113+4948 & S4 0110+49 & $12.48(0.02)$ & $11.37(0.02)$ & $8.43(0.02)$ & $6.12(0.05)$ & $2.26(0.12)$ & $1.37(0.06)$ \\
\hline BZQJ0128+4439 & GB6 J0128+4439 & $14.82(0.04)$ & $13.87(0.04)$ & $11.72(0.21)$ & 8.68() & $2.25(0.13)$ & $0.9(0.1)$ \\
\hline BZQJ0136+4751 & OC 457 & $11.53(0.02)$ & $10.38(0.02)$ & $7.31(0.02)$ & $4.93(0.03)$ & $2.15(0.04)$ & $1.48(0.06)$ \\
\hline BZQJ0205+3212 & B2 $0202+31$ & $14.47(0.04)$ & $12.89(0.03)$ & $9.64(0.05)$ & $7.2(0.1)$ & $2.66(0.14)$ & $2.75(0.08)$ \\
\hline BZQJ0217+7349 & S5 $0212+73$ & $13.99(0.03)$ & $12.9(0.03)$ & $9.56(0.04)$ & $7.21(0.08)$ & $2.82(0.11)$ & $1.3(0.08)$ \\
\hline BZQJ0217+0144 & PKS $0215+015$ & $11.41(0.02)$ & $10.26(0.02)$ & $7.28(0.02)$ & $4.96(0.03)$ & $2.15(0.03)$ & $1.47(0.05)$ \\
\hline BZQJ0230+4032 & B3 $0227+403$ & $13.78(0.03)$ & $12.58(0.03)$ & $9.66(0.04)$ & $7.43(0.12)$ & $2.63(0.06)$ & $1.62(0.07)$ \\
\hline BZQJ0237+2848 & $4 \mathrm{C}+28.07$ & $12.51(0.02)$ & $11.37(0.02)$ & $8.43(0.03)$ & $6.09(0.05)$ & $2.16(0.06)$ & $1.44(0.06)$ \\
\hline BZQJ0245+2405 & B2 $0242+23$ & $15.48(0.05)$ & $14.45(0.07)$ & $11.09(0.14)$ & $8.63(0.34)$ & $2.54(0.08)$ & $1.14(0.15)$ \\
\hline BZQJ0250+1712 & NVSS J025037+171209 & $12.95(0.03)$ & $12.33(0.03)$ & $10.27(0.07)$ & $8.42(0.33)$ & $1.84(0.17)$ & $-0.05(0.06)$ \\
\hline BZQJ0252-2219 & PKS 0250-225 & $14.28(0.03)$ & $13.04(0.03)$ & $9.81(0.05)$ & $7.38(0.12)$ & $2.19(0.05)$ & $1.76(0.07)$ \\
\hline BZQJ0257-1212 & PB 09399 & $14.26(0.03)$ & $13.08(0.03)$ & $10.17(0.05)$ & $8.09(0.17)$ & $2.39(0.14)$ & $1.57(0.07)$ \\
\hline BZQJ0303-7914 & PMN J0303-7914 & $14.28(0.03)$ & $13.06(0.03)$ & $10.02(0.04)$ & $7.53(0.09)$ & $2.2(0.13)$ & $1.7(0.07)$ \\
\hline BZQJ0309+1029 & PKS 0306+102 & $12.26(0.02)$ & $11.15(0.02)$ & $8.12(0.02)$ & $5.78(0.04)$ & $2.26(0.08)$ & $1.39(0.06)$ \\
\hline BZQJ0310+3814 & B3 0307+38 & $14.06(0.04)$ & $12.92(0.03)$ & $9.81(0.05)$ & $7.25(0.1)$ & $2.25(0.16)$ & $1.46(0.08)$ \\
\hline BZQJ0312+0133 & PKS 0310+013 & $13.51(0.03)$ & $12.41(0.03)$ & $9.61(0.04)$ & $7.05(0.08)$ & $2.26(0.08)$ & $1.34(0.06)$ \\
\hline BZQJ0315-1031 & PKS 0313-107 & $15.77(0.06)$ & $14.72(0.08)$ & $11.49(0.18)$ & 9.11() & $2.18(0.13)$ & $1.22(0.17)$ \\
\hline BZQJ0325+2224 & TXS $0322+222$ & $13.68(0.03)$ & $12.42(0.03)$ & $9.16(0.04)$ & $6.75(0.09)$ & $2.41(0.12)$ & $1.82(0.07)$ \\
\hline BZQJ0336+3218 & NRAO 140 & $12.56(0.03)$ & $11.25(0.02)$ & $8.63(0.03)$ & $6.38(0.06)$ & $2.59(0.1)$ & $1.97(0.06)$ \\
\hline BZQJ0339-0146 & PKS 0336-01 & $12.7(0.03)$ & $11.54(0.02)$ & $8.58(0.03)$ & $6.16(0.05)$ & $2.48(0.07)$ & $1.51(0.06)$ \\
\hline BZQJ0348-2749 & PKS 0346-27 & $13.98(0.03)$ & $12.88(0.03)$ & $9.78(0.04)$ & $7.46(0.11)$ & $2.32(0.13)$ & $1.35(0.07)$ \\
\hline BZQJ0349-2102 & PKS 0347-211 & $15.59(0.05)$ & $14.42(0.06)$ & $11.29(0.13)$ & $8.57(0.3)$ & $2.23(0.09)$ & $1.55(0.13)$ \\
\hline BZQJ0402-3147 & PKS 0400-319 & $14.05(0.03)$ & $12.97(0.03)$ & $10.02(0.05)$ & $7.75(0.14)$ & $2.52(0.22)$ & $1.28(0.07)$ \\
\hline BZQJ0403-3605 & PKS 0402-362 & $11.49(0.02)$ & $10.27(0.02)$ & $6.99(0.02)$ & $4.52(0.02)$ & $2.3(0.04)$ & $1.69(0.06)$ \\
\hline BZQJ0405-1308 & PKS 0403-13 & $12.33(0.02)$ & $11.22(0.02)$ & $8.75(0.03)$ & $6.66(0.06)$ & $2.35(0.16)$ & $1.39(0.06)$ \\
\hline BZQJ0413-5332 & PMN J0413-5332 & $15.18(0.04)$ & $14.08(0.04)$ & $11.09(0.08)$ & $8.53(0.23)$ & $2.41(0.09)$ & $1.34(0.09)$ \\
\hline BZQJ0416-1851 & PKS 0414-189 & $14.86(0.04)$ & $13.56(0.04)$ & $10.35(0.06)$ & $7.99(0.17)$ & $2.2(0.09)$ & $1.92(0.09)$ \\
\hline BZQJ0422-0643 & PMN J0422-0643 & $12.56(0.02)$ & $11.58(0.02)$ & $8.78(0.03)$ & $6.54(0.06)$ & $2.39(0.12)$ & $0.99(0.06)$ \\
\hline BZQJ0423-0120 & PKS 0420-01 & $10.84(0.02)$ & $9.72(0.02)$ & $6.62(0.02)$ & $4.25(0.02)$ & $2.3(0.03)$ & $1.41(0.05)$ \\
\hline BZQJ0426+0518 & PKS $0423+051$ & $14.79(0.04)$ & $13.52(0.04)$ & $10.59(0.08)$ & $8.22(0.24)$ & $2.66(0.12)$ & $1.85(0.09)$ \\
\hline BZQJ0438-1251 & PKS 0436-129 & $14.23(0.03)$ & $13.1(0.03)$ & $10.14(0.05)$ & $7.84(0.15)$ & $2.35(0.17)$ & $1.45(0.07)$ \\
\hline BZQJ0442-0017 & PKS 0440-00 & $12.9(0.02)$ & $11.84(0.02)$ & $8.94(0.03)$ & $6.6(0.05)$ & $2.44(0.03)$ & $1.22(0.06)$ \\
\hline BZQJ0448-2109 & PKS 0446-212 & $14.51(0.03)$ & $13.5(0.04)$ & $10.52(0.08)$ & $8.23(0.25)$ & $2.33(0.18)$ & $1.09(0.09)$ \\
\hline BZQJ0453-2807 & PKS 0451-28 & $13.49(0.03)$ & $12.4(0.02)$ & $9.15(0.03)$ & $6.59(0.06)$ & $2.66(0.05)$ & $1.32(0.06)$ \\
\hline BZQJ0455-4615 & PKS 0454-46 & $13.45(0.03)$ & $12.23(0.02)$ & $9.09(0.03)$ & $6.65(0.05)$ & $2.62(0.06)$ & $1.69(0.06)$ \\
\hline BZQJ0456-3136 & PMN J0456-3135 & $14.37(0.03)$ & $13.29(0.03)$ & $10.34(0.05)$ & $7.7(0.11)$ & $2.42(0.14)$ & $1.28(0.07)$ \\
\hline BZQJ0457-2324 & PKS 0454-234 & $12.27(0.02)$ & $11.14(0.02)$ & $8.15(0.02)$ & $5.81(0.03)$ & $2.03(0.02)$ & $1.41(0.06)$ \\
\hline BZQJ0501-0159 & S3 0458-02 & $13.19(0.03)$ & $12.01(0.02)$ & $8.89(0.03)$ & $6.44(0.06)$ & $2.52(0.1)$ & $1.59(0.06)$ \\
\hline BZQJ0502+0609 & PKS 0459+060 & $14.43(0.04)$ & $13.2(0.03)$ & $10.31(0.07)$ & $7.99(0.21)$ & $2.46(0.17)$ & $1.72(0.08)$ \\
\hline BZQJ0505-0419 & S3 0503-04 & $14.93(0.04)$ & $13.81(0.04)$ & $10.52(0.08)$ & $8.41(0.28)$ & $2.21(0.14)$ & $1.38(0.1)$ \\
\hline BZQJ0507-6104 & PMN J0507-6104 & $14.1(0.03)$ & $12.99(0.02)$ & $10.11(0.03)$ & $7.77(0.1)$ & $2.36(0.08)$ & $1.37(0.06)$ \\
\hline BZQJ0509+1011 & PKS 0506+101 & $14.26(0.03)$ & $13.23(0.03)$ & $10.45(0.08)$ & $7.89(0.19)$ & $2.33(0.09)$ & $1.14(0.08)$ \\
\hline BZQJ0510+1800 & PKS 0507+17 & $11.9(0.03)$ & $10.8(0.02)$ & $7.91(0.02)$ & $5.63(0.04)$ & $2.29(0.1)$ & $1.36(0.06)$ \\
\hline BZQJ0515-4556 & PKS 0514-459 & $12.73(0.02)$ & $11.76(0.02)$ & $8.93(0.02)$ & $6.56(0.04)$ & $2.47(0.18)$ & $0.96(0.05)$ \\
\hline BZQJ0526-4830 & PKS 0524-485 & $13.68(0.02)$ & $12.53(0.02)$ & $9.5(0.03)$ & $7.2(0.07)$ & $2.2(0.09)$ & $1.5(0.06)$ \\
\hline BZQJ0530+1331 & PKS $0528+134$ & $14.7(0.04)$ & $13.65(0.04)$ & $10.5(0.09)$ & $7.74(0.16)$ & $2.22(0.09)$ & $1.18(0.1)$ \\
\hline BZQJ0529-0519 & PMN J0529-0519 & $14.3(0.03)$ & $13.42(0.04)$ & $10.19(0.06)$ & $7.83(0.17)$ & $2.3(0.39)$ & $0.71(0.09)$ \\
\hline BZQJ0532-3848 & PMN J0532-3848 & $15.34(0.04)$ & $14.11(0.04)$ & $11.04(0.09)$ & $8.62(0.27)$ & $2.61(0.15)$ & $1.73(0.1)$ \\
\hline BZQJ0532+0732 & OG 050 & $13.62(0.04)$ & $12.42(0.03)$ & $9.24(0.04)$ & $6.7(0.07)$ & $2.31(0.04)$ & $1.64(0.09)$ \\
\hline BZQJ0533+4822 & TXS 0529+483 & $12.7(0.03)$ & $11.45(0.02)$ & $8.26(0.02)$ & $5.8(0.04)$ & $2.31(0.05)$ & $1.78(0.06)$ \\
\hline BZQJ0539-2839 & PKS 0537-286 & $15.79(0.06)$ & $14.75(0.06)$ & $11.41(0.13)$ & $8.59(0.28)$ & $2.83(0.1)$ & $1.18(0.14)$ \\
\hline BZQJ0607-0834 & PKS 0605-08 & $11.94(0.02)$ & $11.36(0.02)$ & $9.06(0.03)$ & $6.81(0.06)$ & $2.36(0.08)$ & $-0.19(0.06)$ \\
\hline BZQJ0610-6058 & PKS 0609-609 & $16.17(0.05)$ & $14.92(0.05)$ & $12.1(0.14)$ & $9.19(0.3)$ & $2.36(0.16)$ & $1.76(0.11)$ \\
\hline
\end{tabular}


Table 1

(Continued)

\begin{tabular}{|c|c|c|c|c|c|c|c|}
\hline ROMA-BZCat Name & 2FGL Name & $m_{1}$ & $m_{2}$ & $m_{3}$ & $m_{4}$ & $\Gamma_{\gamma}$ & $\alpha_{\mathrm{IR}}$ \\
\hline BZQJ0635-7516 & PKS 0637-75 & $11.57(0.02)$ & $10.49(0.02)$ & $7.65(0.02)$ & $5.36(0.03)$ & $2.65(0.06)$ & $1.28(0.05)$ \\
\hline BZQJ0701-4634 & PKS 0700-465 & $13.02(0.03)$ & $11.83(0.02)$ & $8.68(0.02)$ & $6.28(0.05)$ & $2.16(0.12)$ & $1.62(0.06)$ \\
\hline BZQJ0713+1935 & MG2 J071354+1934 & $11.87(0.02)$ & $10.84(0.02)$ & $8.1(0.02)$ & $5.89(0.04)$ & $2.01(0.06)$ & $1.13(0.05)$ \\
\hline BZQJ0725+1425 & $4 \mathrm{C}+14.23$ & $13.42(0.03)$ & $12.31(0.03)$ & $9.43(0.04)$ & $6.98(0.08)$ & $2.04(0.04)$ & $1.37(0.06)$ \\
\hline BZQJ0726+2153 & TXS $0723+220$ & $15.11(0.08)$ & $13.76(0.06)$ & $10.54(0.09)$ & $8.44(0.31)$ & $2.59(0.14)$ & $2.08(0.17)$ \\
\hline BZQJ0726-4728 & PMN J0726-4728 & $14.02(0.04)$ & $12.86(0.03)$ & $9.75(0.04)$ & $7.26(0.08)$ & $2.34(0.09)$ & $1.52(0.07)$ \\
\hline BZQJ0730-1141 & PKS 0727-11 & $11.91(0.02)$ & $10.76(0.02)$ & $7.68(0.02)$ & $5.28(0.04)$ & $2.11(0.02)$ & $1.48(0.05)$ \\
\hline BZQJ0733+5022 & TXS 0730+504 & $13.78(0.03)$ & $12.74(0.03)$ & $9.95(0.05)$ & $7.42(0.1)$ & $2.35(0.12)$ & $1.17(0.07)$ \\
\hline BZQJ0739+0137 & PKS 0736+01 & $11.04(0.02)$ & $10.02(0.02)$ & $7.24(0.02)$ & $5.03(0.03)$ & $2.23(0.08)$ & $1.11(0.05)$ \\
\hline BZQJ0746+2549 & B2 $0743+25$ & $16.11(0.08)$ & $14.9(0.09)$ & $11.39(0.17)$ & $8.48(0.28)$ & $2.85(0.11)$ & $1.66(0.2)$ \\
\hline BZQJ0749+4510 & B3 $0745+453$ & $11.98(0.03)$ & $10.95(0.02)$ & $8.07(0.02)$ & $5.51(0.03)$ & $2.24(0.16)$ & $1.16(0.06)$ \\
\hline BZQJ0750+1231 & OІ 280 & $12.3(0.02)$ & $11.22(0.02)$ & $8.37(0.02)$ & $5.97(0.04)$ & $2.42(0.07)$ & $1.3(0.06)$ \\
\hline BZQJ0805+6144 & TXS 0800+618 & $15.59(0.05)$ & $14.4(0.06)$ & $11.02(0.1)$ & $8.4(0.26)$ & $2.74(0.07)$ & $1.62(0.13)$ \\
\hline BZQJ0808-0751 & PKS 0805-07 & $11.56(0.02)$ & $10.51(0.02)$ & 7.7(0.02) & $5.42(0.03)$ & $1.93(0.03)$ & $1.18(0.05)$ \\
\hline BZQJ0824+3916 & $4 C+39.23$ & $14.18(0.03)$ & $12.95(0.03)$ & $9.75(0.04)$ & 7.24(0.09) & $2.64(0.17)$ & $1.73(0.07)$ \\
\hline BZQJ0824+5552 & OJ 535 & $14.58(0.03)$ & $13.33(0.03)$ & $10.32(0.07)$ & $7.74(0.16)$ & $2.68(0.08)$ & $1.78(0.08)$ \\
\hline BZQJ0830+2410 & S3 $0827+24$ & $13.14(0.03)$ & $11.94(0.02)$ & $8.8(0.03)$ & $6.49(0.06)$ & $2.67(0.07)$ & $1.64(0.06)$ \\
\hline BZQJ0833+4224 & OJ 451 & $12.3(0.02)$ & $11.26(0.02)$ & $8.48(0.03)$ & $6.26(0.05)$ & $2.33(0.13)$ & $1.19(0.06)$ \\
\hline BZQJ0839+0104 & PKS 0837+012 & $14.92(0.04)$ & $13.68(0.04)$ & $10.66(0.09)$ & $8.18(0.25)$ & $2.21(0.11)$ & $1.75(0.1)$ \\
\hline BZQJ0841+7053 & $4 C+71.07$ & $13.73(0.03)$ & $12.59(0.03)$ & $9.16(0.03)$ & $6.68(0.06)$ & $2.95(0.07)$ & $1.47(0.06)$ \\
\hline BZQJ0903+4651 & S4 $0859+47$ & $14.36(0.03)$ & $13.15(0.03)$ & $9.98(0.05)$ & $7.58(0.12)$ & $2.27(0.18)$ & $1.67(0.08)$ \\
\hline BZQJ0912+4126 & B3 $0908+416 B$ & $15.18(0.04)$ & $14.0(0.05)$ & $10.94(0.09)$ & $8.12(0.17)$ & $2.3(0.17)$ & $1.59(0.1)$ \\
\hline BZQJ0916+3854 & S4 0913+39 & $14.57(0.03)$ & $13.43(0.04)$ & $10.5(0.08)$ & $8.39(0.29)$ & $2.53(0.16)$ & $1.47(0.09)$ \\
\hline BZQJ0920+4441 & S4 0917+44 & $13.59(0.03)$ & $12.36(0.03)$ & $9.09(0.03)$ & $6.58(0.07)$ & $2.11(0.03)$ & $1.73(0.07)$ \\
\hline BZQJ0921+6215 & OK 630 & $14.3(0.03)$ & $13.14(0.03)$ & $10.02(0.05)$ & $7.42(0.1)$ & $2.51(0.09)$ & $1.55(0.07)$ \\
\hline BZQJ0937+5008 & GB6 J0937+5008 & $13.19(0.03)$ & $12.02(0.02)$ & $8.91(0.03)$ & $6.4(0.06)$ & $2.5(0.15)$ & $1.55(0.06)$ \\
\hline BZQJ0957+5522 & $4 C+55.17$ & $12.91(0.03)$ & $11.85(0.02)$ & $8.94(0.03)$ & $6.63(0.06)$ & $1.83(0.03)$ & $1.22(0.06)$ \\
\hline BZQJ1044+8054 & S5 1039+81 & $12.44(0.02)$ & $11.33(0.02)$ & $8.42(0.02)$ & $6.12(0.04)$ & $2.54(0.15)$ & $1.39(0.06)$ \\
\hline BZQJ1056+7011 & S5 $1053+70$ & $14.86(0.03)$ & $13.62(0.03)$ & $10.22(0.05)$ & $7.7(0.12)$ & $2.64(0.1)$ & $1.74(0.08)$ \\
\hline BZQJ1058+8114 & S5 1053+81 & $13.37(0.03)$ & $12.2(0.02)$ & $9.04(0.03)$ & $6.64(0.06)$ & $2.58(0.09)$ & $1.57(0.06)$ \\
\hline BZQJ1258-2219 & PKS $1256-220$ & $12.85(0.03)$ & $11.65(0.03)$ & $8.72(0.03)$ & $6.55(0.07)$ & $2.3(0.07)$ & $1.61(0.06)$ \\
\hline BZQJ1316-3338 & PKS 1313-333 & $12.77(0.03)$ & $11.65(0.02)$ & $8.68(0.03)$ & $6.34(0.06)$ & $2.31(0.06)$ & $1.41(0.06)$ \\
\hline BZQJ1332-1256 & PMN J1332-1256 & $15.51(0.06)$ & $14.29(0.07)$ & $11.36(0.22)$ & $8.1(0.27)$ & $2.38(0.04)$ & $1.69(0.16)$ \\
\hline BZQJ1337-1257 & PKS 1335-127 & $12.6(0.03)$ & $11.39(0.02)$ & $8.2(0.02)$ & $5.65(0.04)$ & $2.44(0.07)$ & $1.67(0.06)$ \\
\hline BZQJ1342-2051 & PKS B1339-206 & $14.72(0.04)$ & $13.26(0.04)$ & $10.05(0.06)$ & $7.58(0.14)$ & $2.63(0.13)$ & $2.4(0.09)$ \\
\hline BZQJ1344-1723 & PMN J1344-1723 & $13.33(0.03)$ & $12.19(0.03)$ & $9.15(0.04)$ & $6.9(0.09)$ & $1.95(0.06)$ & $1.47(0.06)$ \\
\hline BZQJ1347-3750 & PMN J1347-3750 & $14.47(0.04)$ & $13.32(0.04)$ & $10.33(0.06)$ & $7.94(0.17)$ & $2.32(0.12)$ & $1.46(0.09)$ \\
\hline BZQJ1351+0031 & PKS 1348+007 & $13.94(0.03)$ & $12.89(0.03)$ & $10.15(0.07)$ & $8.48(0.3)$ & $2.29(0.11)$ & $1.21(0.08)$ \\
\hline BZQJ1354-1041 & PKS 1352-104 & $13.42(0.03)$ & $12.4(0.03)$ & $9.3(0.04)$ & $6.96(0.09)$ & $2.57(0.08)$ & $1.09(0.07)$ \\
\hline BZQJ1357+7643 & S5 $1357+76$ & $14.95(0.03)$ & $13.76(0.03)$ & $10.79(0.06)$ & $7.99(0.13)$ & $2.3(0.1)$ & $1.63(0.07)$ \\
\hline BZQJ1408-0752 & PKS B1406-076 & $13.84(0.03)$ & $12.54(0.03)$ & $9.52(0.05)$ & $7.2(0.13)$ & $2.43(0.06)$ & $1.93(0.07)$ \\
\hline BZQJ1427-4206 & PKS B1424-418 & $11.26(0.02)$ & $10.17(0.02)$ & $7.22(0.02)$ & $4.94(0.03)$ & $1.96(0.03)$ & $1.32(0.05)$ \\
\hline BZQJ1436+2321 & PKS B1434+235 & $14.06(0.03)$ & $12.79(0.03)$ & $9.49(0.04)$ & $6.86(0.07)$ & $2.41(0.18)$ & $1.84(0.07)$ \\
\hline BZQJ1441-3303 & PKS 1438-328 & $14.17(0.03)$ & $13.04(0.04)$ & $10.01(0.06)$ & $7.62(0.15)$ & $2.76(0.17)$ & $1.44(0.08)$ \\
\hline BZQJ1443+2501 & PKS $1441+25$ & $15.41(0.05)$ & $14.34(0.06)$ & $11.29(0.16)$ & $8.73(0.4)$ & $2.03(0.12)$ & $1.25(0.13)$ \\
\hline BZQJ1457-3539 & PKS 1454-354 & $13.0(0.03)$ & $11.92(0.02)$ & $9.07(0.03)$ & $6.89(0.07)$ & $2.11(0.03)$ & $1.28(0.06)$ \\
\hline BZQJ1504+1029 & PKS $1502+106$ & $13.21(0.03)$ & $12.05(0.02)$ & $9.06(0.03)$ & $6.62(0.06)$ & $2.15(0.02)$ & $1.54(0.06)$ \\
\hline BZQJ1505+0326 & PKS $1502+036$ & $13.94(0.03)$ & $12.95(0.03)$ & $9.68(0.04)$ & $7.03(0.09)$ & $2.51(0.07)$ & $1.0(0.07)$ \\
\hline BZQJ1506+3730 & B2 1504+37 & $13.9(0.03)$ & $12.54(0.03)$ & $9.56(0.04)$ & $7.45(0.1)$ & $2.57(0.1)$ & $2.09(0.07)$ \\
\hline BZQJ1509-4340 & PMN J1509-4340 & $14.27(0.05)$ & $13.14(0.04)$ & $10.5(0.07)$ & $7.74(0.14)$ & $2.65(0.14)$ & $1.44(0.11)$ \\
\hline BZQJ1510-0543 & PKS 1508-05 & $13.18(0.02)$ & $12.01(0.02)$ & $9.06(0.03)$ & $6.59(0.07)$ & $2.44(0.06)$ & $1.55(0.06)$ \\
\hline BZQJ1512-0905 & PKS 1510-08 & $11.27(0.02)$ & $10.17(0.02)$ & $7.37(0.02)$ & $5.06(0.03)$ & $2.29(0.01)$ & $1.32(0.06)$ \\
\hline BZQJ1520+4211 & B3 $1518+423$ & $13.15(0.02)$ & $12.21(0.02)$ & $9.48(0.03)$ & 7.3(0.09) & $2.5(0.31)$ & $0.88(0.06)$ \\
\hline BZQJ1521+4336 & B3 $1520+437$ & $15.62(0.05)$ & $14.5(0.06)$ & $10.98(0.1)$ & $8.74(0.31)$ & $2.99(0.16)$ & $1.4(0.13)$ \\
\hline BZQJ1539+2744 & MG2 J153938+2744 & $14.17(0.03)$ & $12.95(0.03)$ & $9.86(0.04)$ & $7.41(0.1)$ & $1.99(0.13)$ & $1.68(0.07)$ \\
\hline BZQJ1549+0237 & PKS $1546+027$ & $12.21(0.02)$ & $11.13(0.02)$ & $8.47(0.03)$ & $6.22(0.05)$ & $2.46(0.07)$ & $1.27(0.06)$ \\
\hline BZQJ1550+0527 & $4 \mathrm{C}+05.64$ & $13.38(0.03)$ & $12.2(0.02)$ & $9.1(0.03)$ & $6.61(0.07)$ & $2.32(0.11)$ & $1.6(0.06)$ \\
\hline BZQJ1608+1029 & $4 \mathrm{C}+10.45$ & $12.56(0.03)$ & $11.46(0.02)$ & $8.6(0.03)$ & $6.23(0.04)$ & $2.33(0.1)$ & $1.34(0.06)$ \\
\hline BZQJ1610-3958 & PMN J1610-3958 & $11.98(0.03)$ & $10.87(0.02)$ & $7.95(0.02)$ & $5.58(0.03)$ & $2.61(0.1)$ & $1.36(0.07)$ \\
\hline BZQJ1613+3412 & OS 319 & $14.02(0.03)$ & $12.89(0.03)$ & $9.91(0.04)$ & $7.38(0.1)$ & $2.31(0.17)$ & $1.44(0.07)$ \\
\hline BZQJ1617-7717 & PKS 1610-77 & $13.23(0.03)$ & $12.16(0.03)$ & $9.07(0.03)$ & $6.62(0.06)$ & $2.5(0.05)$ & $1.27(0.06)$ \\
\hline BZQJ1635+3808 & $4 \mathrm{C}+38.41$ & $13.09(0.03)$ & $11.86(0.02)$ & $8.51(0.02)$ & $5.96(0.04)$ & $2.25(0.03)$ & $1.71(0.06)$ \\
\hline BZQJ1637+4717 & $4 \mathrm{C}+47.44$ & $12.63(0.02)$ & $11.45(0.02)$ & $8.35(0.02)$ & $5.98(0.04)$ & $2.41(0.06)$ & $1.59(0.06)$ \\
\hline BZQJ1640+3946 & NRAO 512 & $15.0(0.04)$ & $13.81(0.04)$ & $10.72(0.07)$ & $8.26(0.19)$ & $2.36(0.06)$ & $1.6(0.08)$ \\
\hline BZQJ1642+3948 & $3 C 345$ & $11.8(0.03)$ & $10.63(0.02)$ & $7.52(0.02)$ & $5.13(0.03)$ & $2.49(0.06)$ & $1.54(0.06)$ \\
\hline BZQJ1656+6012 & 87GB 165604.4+601702 & $14.26(0.03)$ & $13.26(0.03)$ & $10.62(0.05)$ & $8.18(0.14)$ & $2.36(0.21)$ & $1.03(0.06)$ \\
\hline
\end{tabular}


Table 1

(Continued)

\begin{tabular}{|c|c|c|c|c|c|c|c|}
\hline ROMA-BZCat Name & 2FGL Name & $m_{1}$ & $m_{2}$ & $m_{3}$ & $m_{4}$ & $\Gamma_{\gamma}$ & $\alpha_{\mathrm{IR}}$ \\
\hline BZQJ1703-6212 & CGRaBS J1703-6212 & $12.27(0.03)$ & $11.26(0.02)$ & $8.44(0.02)$ & $6.19(0.04)$ & $2.43(0.04)$ & $1.07(0.06)$ \\
\hline BZQJ1709+4318 & B3 $1708+433$ & $13.5(0.03)$ & $12.35(0.02)$ & $9.31(0.03)$ & $6.98(0.07)$ & $2.31(0.05)$ & $1.48(0.06)$ \\
\hline BZQJ1722+1013 & TXS $1720+102$ & $12.42(0.02)$ & $11.33(0.02)$ & $8.33(0.02)$ & $5.98(0.04)$ & $2.23(0.06)$ & $1.31(0.06)$ \\
\hline BZQJ1724+4004 & S4 $1722+40$ & $14.16(0.03)$ & $13.09(0.03)$ & $10.0(0.04)$ & $7.66(0.1)$ & $2.34(0.06)$ & $1.25(0.06)$ \\
\hline BZQJ1727+4530 & S4 $1726+45$ & $13.67(0.02)$ & $12.51(0.02)$ & $9.37(0.03)$ & $6.82(0.06)$ & $2.58(0.06)$ & $1.52(0.06)$ \\
\hline BZQJ1728+1215 & PKS $1725+123$ & $13.66(0.03)$ & $12.48(0.03)$ & $9.42(0.04)$ & $6.98(0.08)$ & $2.09(0.2)$ & $1.58(0.06)$ \\
\hline BZQJ1728+0427 & PKS $1725+044$ & $12.3(0.02)$ & $11.31(0.02)$ & $8.45(0.03)$ & $5.87(0.04)$ & $2.53(0.08)$ & $1.02(0.06)$ \\
\hline BZQJ1730+0024 & PKS 1728+004 & $12.85(0.03)$ & $11.72(0.03)$ & $8.78(0.03)$ & $6.49(0.07)$ & $2.31(0.07)$ & $1.43(0.06)$ \\
\hline BZQJ1733-1304 & PKS 1730-13 & $12.14(0.03)$ & $11.06(0.02)$ & $8.1(0.02)$ & $5.66(0.04)$ & $2.24(0.09)$ & $1.3(0.06)$ \\
\hline BZQJ1734+3857 & B2 $1732+38 \mathrm{~A}$ & $13.49(0.03)$ & $12.31(0.02)$ & $9.15(0.03)$ & $6.79(0.06)$ & $2.24(0.04)$ & $1.58(0.06)$ \\
\hline BZQJ1739+4955 & S4 1738+49 & $12.61(0.03)$ & $11.5(0.02)$ & $8.64(0.02)$ & $6.37(0.04)$ & $2.2(0.09)$ & $1.36(0.06)$ \\
\hline BZQJ1740+5211 & $4 C+51.37$ & $12.33(0.02)$ & $11.16(0.02)$ & $8.16(0.02)$ & $5.89(0.03)$ & $2.5(0.04)$ & $1.54(0.05)$ \\
\hline BZQJ1745+2252 & TXS $1742+228$ & $15.21(0.04)$ & $14.05(0.05)$ & $11.08(0.1)$ & $8.55(0.28)$ & $2.87(0.17)$ & $1.52(0.11)$ \\
\hline BZQJ1801+4404 & S4 $1800+44$ & $13.23(0.02)$ & $12.19(0.02)$ & $9.16(0.03)$ & $6.72(0.05)$ & $2.66(0.14)$ & $1.16(0.06)$ \\
\hline BZQJ1818+0903 & MG1 J181841+0903 & $13.08(0.03)$ & $11.98(0.03)$ & $9.11(0.03)$ & $6.82(0.08)$ & $2.32(0.08)$ & $1.32(0.07)$ \\
\hline BZQJ1848+3219 & B2 $1846+32 \mathrm{~A}$ & $13.36(0.03)$ & $12.19(0.02)$ & $9.13(0.03)$ & $6.69(0.06)$ & $2.38(0.09)$ & $1.54(0.06)$ \\
\hline BZQJ1852+4855 & S4 $1851+48$ & $13.08(0.03)$ & $12.0(0.02)$ & $9.13(0.02)$ & $6.89(0.06)$ & $2.28(0.04)$ & $1.28(0.06)$ \\
\hline BZQJ1903-6749 & PMN J1903-6749 & $13.46(0.03)$ & $12.38(0.02)$ & $9.49(0.03)$ & $7.08(0.08)$ & $2.49(0.1)$ & $1.3(0.06)$ \\
\hline BZQJ1911-2006 & PKS B1908-201 & $11.27(0.02)$ & $10.17(0.02)$ & $7.16(0.03)$ & () & $2.21(0.05)$ & $1.36(0.06)$ \\
\hline BZQJ1923-2104 & TXS 1920-211 & $11.09(0.02)$ & $10.08(0.02)$ & $7.33(0.02)$ & $5.02(0.03)$ & $2.1(0.04)$ & $1.08(0.05)$ \\
\hline BZQJ1924-2914 & PKS B1921-293 & $10.81(0.02)$ & $9.65(0.02)$ & $6.53(0.02)$ & $4.1(0.02)$ & $2.43(0.05)$ & $1.52(0.06)$ \\
\hline BZQJ1954-1123 & TXS 1951-115 & $13.68(0.04)$ & $12.5(0.03)$ & $9.45(0.05)$ & $7.08(0.12)$ & $2.25(0.05)$ & $1.57(0.08)$ \\
\hline BZQJ1957-3845 & PKS 1954-388 & $12.35(0.02)$ & $11.19(0.02)$ & $8.28(0.02)$ & $5.87(0.04)$ & $2.36(0.05)$ & $1.51(0.05)$ \\
\hline BZQJ1959-4246 & PMN J1959-4246 & $13.62(0.03)$ & $12.45(0.03)$ & $9.48(0.04)$ & 7.01(0.08) & $2.41(0.05)$ & $1.53(0.07)$ \\
\hline BZQJ2007-4434 & PKS 2004-447 & $13.37(0.03)$ & $12.28(0.03)$ & $9.42(0.04)$ & 7.01(0.09) & $2.47(0.12)$ & $1.3(0.06)$ \\
\hline BZQJ2023-1139 & PMN J2023-1140 & $15.38(0.05)$ & $14.63(0.08)$ & $11.24(0.17)$ & $7.96(0.21)$ & $2.07(0.11)$ & $0.32(0.16)$ \\
\hline BZQJ2025-0735 & PKS 2023-07 & $13.32(0.03)$ & $12.08(0.02)$ & $8.87(0.03)$ & $6.33(0.06)$ & $2.15(0.03)$ & $1.75(0.06)$ \\
\hline BZQJ2030-0622 & TXS 2027-065 & $14.15(0.03)$ & $12.93(0.03)$ & $9.8(0.06)$ & $8.03(0.26)$ & $2.73(0.1)$ & $1.7(0.08)$ \\
\hline BZQJ2056-4714 & PKS 2052-47 & $12.6(0.03)$ & $11.31(0.02)$ & $7.99(0.02)$ & $5.54(0.04)$ & $2.23(0.04)$ & $1.89(0.06)$ \\
\hline BZQJ2135-5006 & PMN J2135-5006 & $16.0(0.08)$ & $15.0(0.11)$ & $11.53(0.21)$ & 9.01() & $2.58(0.1)$ & $1.05(0.23)$ \\
\hline BZQJ2147-7536 & PKS 2142-75 & $11.01(0.02)$ & $9.78(0.02)$ & $6.61(0.02)$ & $4.23(0.02)$ & $2.52(0.04)$ & $1.72(0.05)$ \\
\hline BZQJ2202-8338 & PKS 2155-83 & $13.82(0.03)$ & $12.67(0.02)$ & $9.7(0.04)$ & $7.46(0.1)$ & $2.2(0.08)$ & $1.48(0.06)$ \\
\hline
\end{tabular}

Notes. Column 1 contains the name of the blazer in the ROMA-BZCat catalog; Column 2: the name of the associated source from the 2FGL catalog; Columns 3-6: the values of the magnitude in the four WISE filters ([3.4], [4.6], [12], and [22] $\mu \mathrm{m}$, respectively); Column 7: the $\Gamma \gamma$ photon index of the sources from the 2FGL catalog; and Column 8: the IR spectral index $\alpha_{12}$ evaluated from WISE magnitudes ([3.4] and [4.6]). Errors are reported in parentheses.

\section{REFERENCES}

Abdo, A. A., Ackermann, M., Agudo, I., et al. 2010, ApJ, 716, 30 Ackermann, M., Ajello, M., Allafort, A., et al. 2011, ApJ, 743, 171

Blandford, R. D., \& Rees, M. J. 1978, in BL Lac Objects (Pittsburgh, PA: Univ. Pittsburgh Press), 328

Cavaliere, A., \& D'Elia, V. 2002, ApJ, 571, 226

Chen, P. S., Fu, H. W., \& Gao, Y. F. 2005, New Astron., 11, 27

Cutri, R. M., Wright, E. L., Conrow, T., et al. 2011, Explanatory Supplement to the WISE Preliminary Data Release Products, 1, http://wise2.ipac.caltech. edu/docs/release/prelim/expsup/wise_prelrel_toc.html

Dermer, C. D., \& Schlickeiser, R. 2002, ApJ, 575, 667

Giommi, P., Padovani, P., Polenta, G., et al. 2011, arXiv:1110.4706

Giommi, P., Piranomonte, S., Perri, M., \& Padovani, P. 2005, A\&A, 434, 385

Giroletti, M., Reimer, A., Fuhrmann, L., \& Pavlidou, V. 2010, Accretion and Ejection in AGN: A Global View, 427, 283

Inoue, S., \& Takahara, F. 1996, ApJ, 463, 555

Kovalev, Y. Y., Aller, H. D., Aller, M. F., et al. 2009, ApJ, 696, L17
León-Tavares, J., Valtaoja, E., Chavushyan, V. H., et al. 2011, MNRAS, 411, 1127

Marscher, A. P., \& Gear, W. K. 1985, ApJ, 298, 114

Maselli, A., Cusumano, G., Massaro, E., et al. 2011, A\&A, 531, A153

Massaro, E., Giommi, P., Leto, C., et al. 2009, A\&A, 495, 691

Massaro, E., Giommi, P., Leto, C., et al. 2010, arXiv:1006.0922

Massaro, F., D’Abrusco, R., Ajello, M., Grindlay, J. E., \& Smith, H. A. 2011a, ApJ, 740, L48

Massaro, F., Paggi, A., Elvis, M., \& Cavaliere, A. 2011b, ApJ, 739, 73

Massaro, F., et al. 2011c, ApJ, submitted

Padovani, P., \& Giommi, P. 1995, MNRAS, 277, 1477

Padovani, P., Perlman, E. S., Landt, H., Giommi, P., \& Perri, M. 2003, ApJ, 588, 128

Taylor, M. B. 2005, in ASP Conf. Ser. 347, Astronomical Data Analysis Software and Systems XIV, ed. P. Shopbell, M. Britton, \& R. Ebert (San Francisco, CA: ASP), 29

The Fermi-LAT Collaboration 2011, ApJ, submitted (arXiv:1108.1435)

Wright, E. L., Eisenhardt, P. R. M., Mainzer, A. K., et al. 2010, AJ, 140, 1868 